\title{
Pulsed Photonuclear Assessment (PPA) Technology Enhancement Study
}

J.L. Jones

B.W. Blackburn

K.J. Haskell

J.T. Johnson

D.R. Norman

J.W. Sterbentz

S.M. Watson

J.F. Harmon

A.W. Hunt

April 2006

The INL is a U.S. Department of Energy National Laboratory operated by Battelle Energy Alliance 
INL/EXT-06-11175

\title{
Pulsed Photonuclear Assessment (PPA) Technology Enhancement Study
}

\author{
J.L. Jones ${ }^{a}$ \\ B.W. Blackburn ${ }^{a}$ \\ K.J. Haskella \\ J.T. Johnson ${ }^{a}$ \\ D.R. Norman ${ }^{a}$ \\ J.W. Sterbentz ${ }^{a}$ \\ S.M. Watson ${ }^{\mathrm{a}}$ \\ J.F. Harmon ${ }^{b}$ \\ A.W. Hunt ${ }^{\text {b }}$
}

${ }^{\mathrm{a}}$ INL

${ }^{\mathrm{b}}$ ISU

April 2006

Idaho National Laboratory

Idaho Falls, Idaho 83415

Prepared for the

U.S. Department of Homeland Security Office of Domestic Nuclear Detection Under DOE Idaho Operations Office

Contract DE-AC07-05ID14517 


\begin{abstract}
The Idaho National Laboratory (INL) along with the Los Alamos National Laboratory (LANL) and Idaho State University's Idaho Accelerator Center (IAC) has designed and tested a nominal $10-\mathrm{MeV}$ prototype Pulsed Photonuclear Assessment (PPA) inspection system to detect shielded nuclear material. This report highlights two specific areas that will provide further PPA technology enhancements, namely, an optimal gamma-ray detection system and the off-axis radiation detection sensitivity.

Detection of low-atomic number $(\mathrm{Z})$ shielded nuclear material had been initially addressed by the inclusion of dedicated Geiger-Müller (GM) detectors co-located above each of the Photonuclear Neutron Detectors (PNDs). Several different radiation detectors were investigated to assess if this type of gammaray detector was optimal. The LND 719 GM detector was shown to have the best photon sensitivity and demonstrated an optimal ability to detect low-Z shielded nuclear material. Beyond the technical performance of this detector, its low cost and availability makes it a logical choice for a field-deployable system.

In terms of off-axis detection sensitivity, simulation and benchmarking experiments have indicated that the PPA inspection system can successfully detect nuclear material (within 120 seconds) in various shielding configurations even when it is located at a distance of as much as $30 \mathrm{~cm}$ off the interrogating beam axis (the exact sensitivity to off-axis interrogations will be largely dependent on the actual shielding material). As a general rule, high- $Z$ shielding will allow detection at larger off-axis distances than low-Z materials.
\end{abstract}




\section{CONTENTS}

Abstract iii

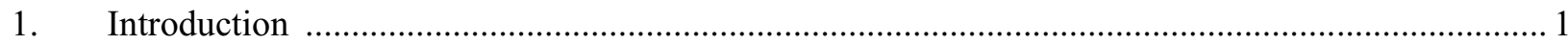

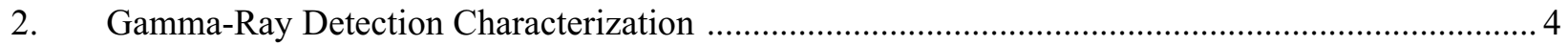

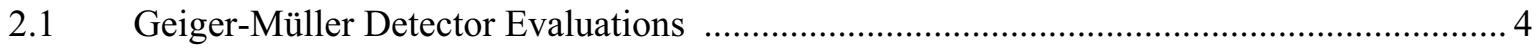

2.1.1 Gm Detectors In The Flash …................................................................... 5

2.1.2 Gm Detector Voltage And Dead Time Performance ....................................... 5

2.2 Neutron And Gamma Detector Normalization Factors …........................................... 7

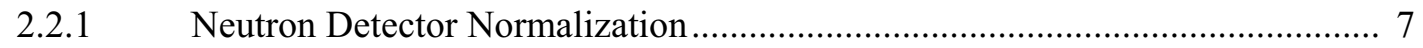

2.2.2 Gamma-Ray Detector Normalization.......................................................... 8

2.2.3 Gm Detection Figure-Of-Merit Algorithm ................................................ 9

2.2.4 Gamma-Ray Signatures Versus Selected Regions-Of-Interest ........................ 10

2.3 Gamma Spectra Utilizing High-Purity Germanium Detectors ....................................... 10

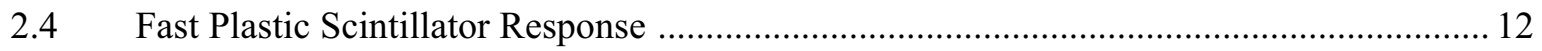

2.4.1 Fast Plastic Experimental Configuration ….................................................... 12

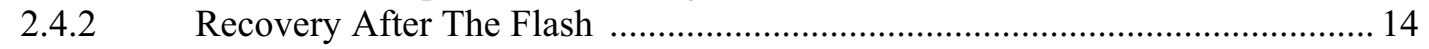

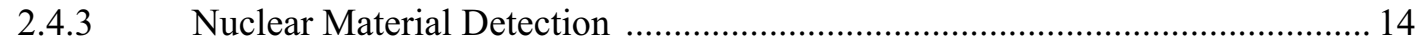

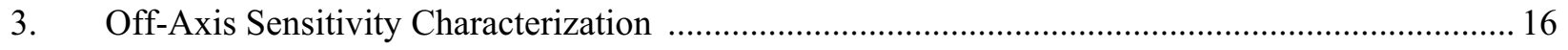

3.1 Numerical Simulations And Experimental Benchmarks ............................................... 16

3.1.1 Experimental Setup For Benchmark Testing …........................................ 16

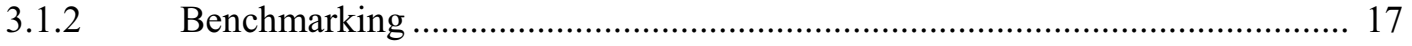

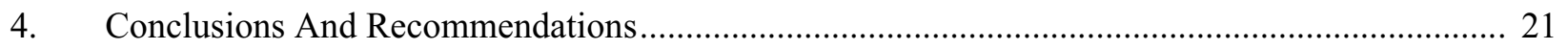

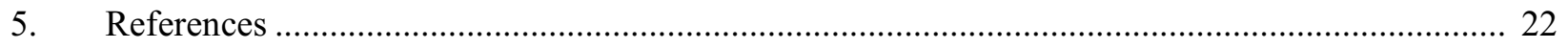

Appendix A Geiger-Müller (GM) Experimental Descriptions ........................................................ 24

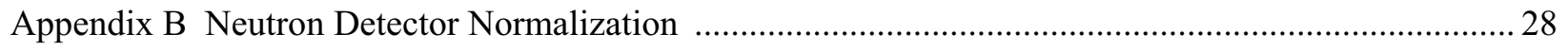

Appendix C Gamma-Ray Detector Normalization ....................................................................... 32

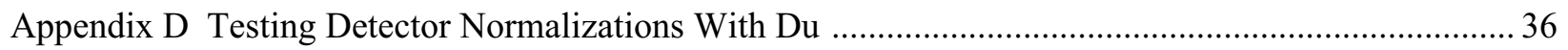




\section{FIGURES}

Figure 1. Schematic of the PPA prototype nuclear material detection/inspection system......................... 1

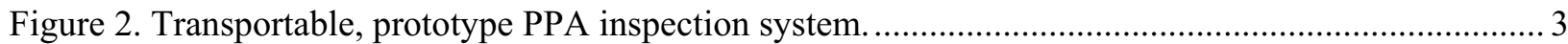

Figure 3. Cargo configuration schematic top view (left) and end view (right). The detector location numbers are shown in the "end view." ......................................................................................... 3

Figure 4. MCS data collected for GM detector models LND 719 and LND 740 during a photon

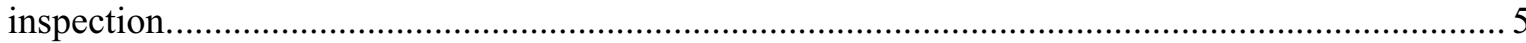

Figure 5. Voltage plateau curves and dead time responses for selected LND Gamma-Ray detectors. ....... 6

Figure 6. Multiple investigations of raw PND data (top), normalized PND data (center), and PND

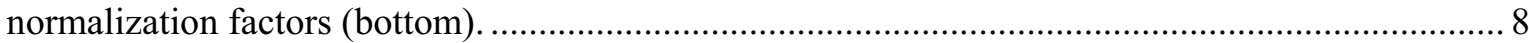

Figure 7. Raw GM data (top), normalized GM data (center), and GM normalization factors (bottom)...... 9

Figure 8. Background without accelerator operations, with and without DU....................................... 11

Figure 9. Gamma-ray spectra for a $10-\mathrm{MeV}, 125-\mathrm{Hz}$ Linac operation with and without DU.................. 12

Figure 10. Plan-view illustration of experimental setup. Note that the lead shield completely surrounds the entire detector with the exception of the front and rear faces

Figure 11. Photographs of experimental setup illustrating relative positions of the polyethylene pallet and shielded detector assembly (left side view is from Polyethylene Calibration Pallet)

Figure 12. Time response of scintillator and GM detectors immediately after the flash.

Figure 13. Ratio of the FOM for the polyethylene pallet (DU/no DU)

Figure 14. Illustration of beam off-axis experiment geometry.

Figure 15. Plan view off-axis experiment geometry.

Figure 16. Photon dose rate as a function of off-axis distance for the DU billet at $2.5-\mathrm{m}$. 18

Figure 17. Photon dose rate as a function of off-axis distance for the DU billet at 4.9-m. 18

Figure 18. Normalized delayed neutron counts versus perpendicular off-axis distance of the DU billet at a

2.5-meter photon converter-to-DU distance.

Figure 19. Normalized delayed neutron counts versus perpendicular off-axis distance of the DU billet at a

4.9-meter photon converter-to-DU distance.

Figure 20. Convolution of experimental pallet data with off-axis MCNPX simulations. 20

Figure B-1. PND test data (top) with calculated correction counts (bottom). 30 
Figure B-2. Accelerator effects on corrected PND responses with accelerator ON (top) and OFF (bottom).

Figure C-1. GM detector raw data with accelerator ON (top), calculated correction factors (center), and raw data with accelerator OFF (bottom).

Figure D-1. Raw data (top) for GM detectors with and without DU and calculated correction factors (bottom) applied with and without DU 38

Figure D-2. Raw data (top) for PND detectors with and without DU and calculated correction factors (bottom) applied with and without DU. 


\section{Pulsed Photonuclear Assessment (PPA) Technology Enhancement Study}

\section{INTRODUCTION}

For several decades, researchers at the Idaho National Laboratory (INL) and Idaho State University's Idaho Accelerator Center (IAC) have applied energetic photon interrogation methods to support treaty verification applications related to nuclear weapon configurations. These methods have required the development of enhanced radiation detectors ${ }^{1-5}$ and the design and fabrication of a unique, transportable, energy-selectable electron accelerator- the INL Varitron. ${ }^{6}$ The recent development of the Pulsed Photonuclear Assessment (PPA) technique, a collaboration with Los Alamos National Laboratory (LANL) and the IAC has continued to support nuclear material detection in various challenging (shielded) configurations. ${ }^{7}$ Currently, the Department of Homeland Security's (DHS) Science and Technology Directorate and the Domestic Nuclear Detection Office (DNDO) have fostered the development of an advanced nuclear material detection capability applicable to a wide variety of cargo-container types and loading configurations. ${ }^{8-15}$ While this report's predecessor ${ }^{8}$ identified and described the development of key detection components of the proposed 10-MeV PPA prototype inspection system, this follow-up report focuses on two specific recommendations made in the CY05 report ${ }^{15}$, namely: (1) enhancement of the gamma detection system, (2) assessment of the off-axis detection sensitivity. Figure 1 is a schematic of the current prototype PPA inspection system for shielded nuclear material detection.

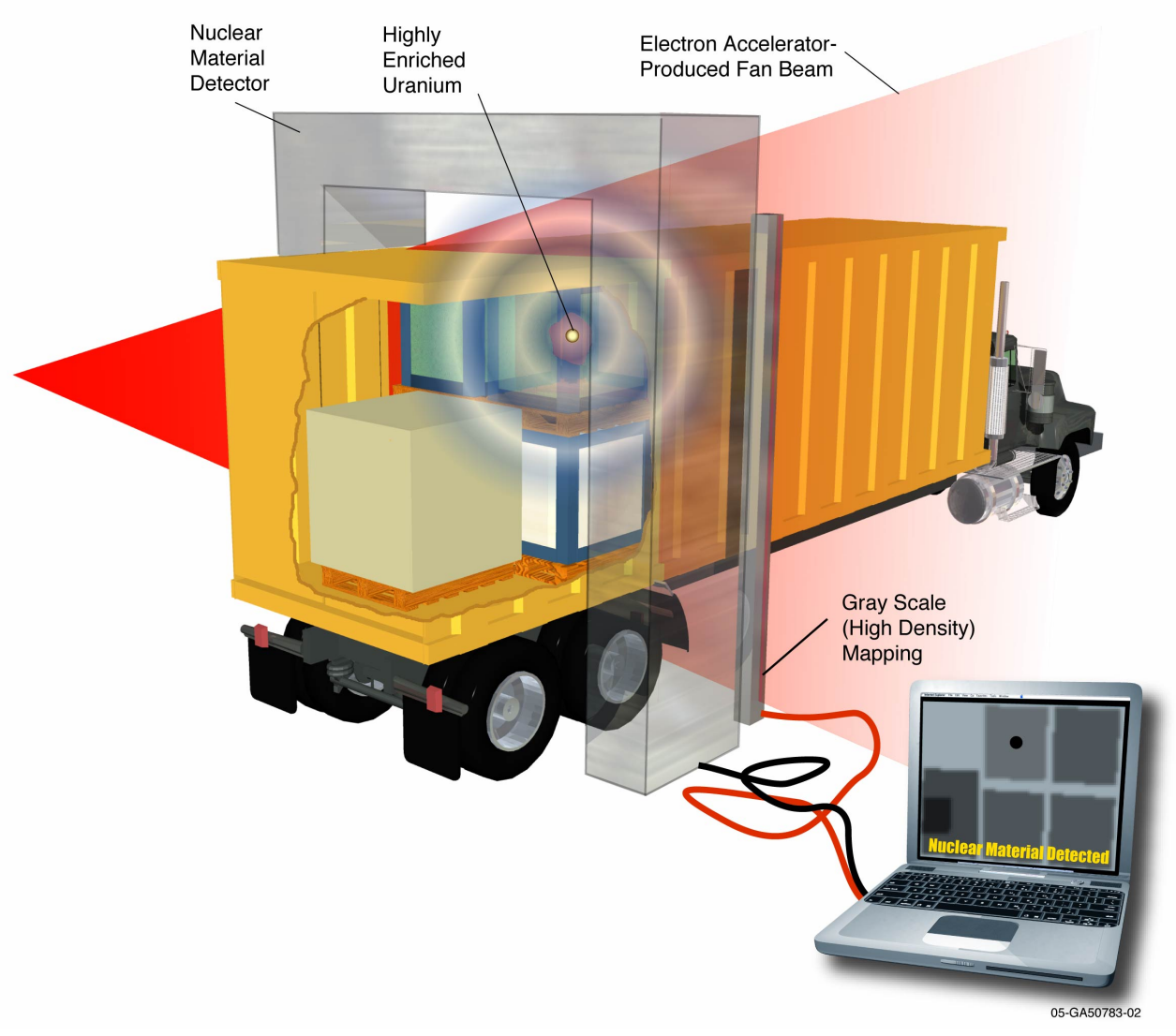

Figure 1. Schematic of the PPA prototype nuclear material detection/inspection system. 
The first recommendation focused on an investigation into a possible enhancement of the original PPA gamma-ray detection system in order to utilize photon signatures to detect nuclear material within hydrogenous type shielding. Geiger-Müller (GM) tubes were initially selected for use in the PPA system as an inexpensive gamma-ray detection method to complement the optimized neutron-based detection system. As indicated in the CY-05 report, simple GM detectors, utilizing preliminary detection algorithms, demonstrated that it was feasible to utilize gamma-ray counting as an indicator of nuclear material within low-atomic number $(Z)$ shielding. While these simple detectors have indicated their viability within the challenging pulsed photonuclear inspection environment, the detection methodology required optimization. This optimization process prompted the following assessments:

1. Performance evaluation of several different types of GM detectors.

2. Thermal neutron correction algorithms to enhance detection probabilities based on GM response.

3. Performance evaluation of an INL-modified HPGe detector and fast plastic scintillator.

The second proposed recommendation addressed the off-beam axis detection capability of the 10$\mathrm{MeV}$ PPA system. This type of characterization is essential for any field-deployable system to address applicability to primary or secondary inspection screening. Determination of interrogation parameters such as the number of beams, collimation (fan or cone), beam current, and dose to cargo will depend on the extent of off-axis sensitivity. In order to characterize the off-axis detection sensitivity, numeric simulations for selected configurations were performed with MCNPX. These numeric studies were experimentally benchmarked in order to quantify the extent to which the PPA system can detect nuclear material when it is placed in positions which are not directly along the photon-producing, electron beam axis.

The most recent version of the nominal $10-\mathrm{MeV}$ transportable, prototype nuclear material detection system is shown in Figure 2. It utilizes arrays of custom-built and commercially-available Photonuclear Neutron Detectors (PND) ${ }^{1}$ [patented design using 10-atm., helium-3 (He-3) tubes with unique electronics and shielding] and co-located, unshielded GM detectors [patent-pending, LND, Inc. tubes (Model 719) with electronics and acquisition software]. Nine pairs of collocated GM and PND detectors are horizontally mounted on two transportable detector assemblies. Also shown in Figure 2 is a representative inspection application utilizing a one-and-a-half metric-ton cargo (i.e., wood and polyethylene pallet loading) configuration positioned under the detector array. Inside the trailer, located behind the detectors and the pallets, are the other major components of the prototype PPA system including the pulsed photon source and the data acquisition system. User controlled operation is provided from a remote location. 


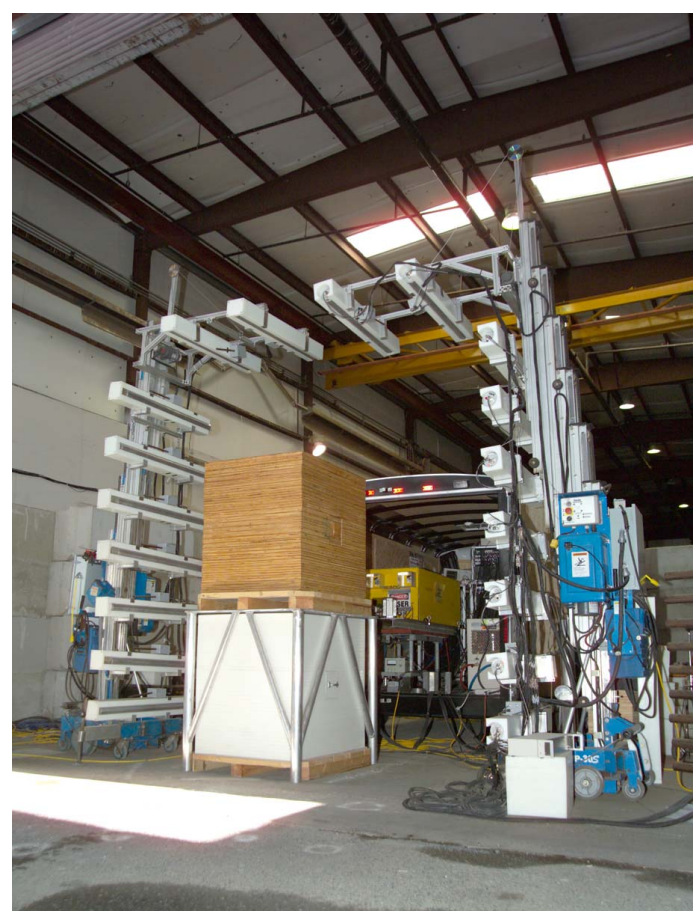

Figure 2. Transportable, prototype PPA inspection system.

This prototype system has been shown to detect nuclear material in a series of seven INL Calibration Pallets ${ }^{8}$ even when these pallets are placed in a "challenging" location within a realistic cargo container configuration ${ }^{14}$. The "challenging" inspection configuration is identified in Figure 3. Note the position locations of detectors \#3 and \#16 (Figure 3; right) are on the prototype accelerator beam center line and a $19^{\text {th }}$ PND (not shown) is typically located under the test configuration when deployed. The average of four additional polyethylene-shielded PNDs, located with the PND detector assemblies, are used to measure background neutrons. The nuclear material is placed in the center of a pallet and the pallet is positioned in the center of the cargo container. This inspection scenario represents a "challenge" because it provides both a realistic photon source-to-cargo center distance ( $\sim 2.5$ to $3 \mathrm{~m})$ while maximizing the nuclear material-to-detector distances for all PND and GM detectors. For this selected cargo inspection configuration, the interrogating photon beam travels through the cargo at nearly a 45-degree angle and the radiation detectors are positioned along the sides of the cargo container (no detectors are placed below the container in this inspection configuration).
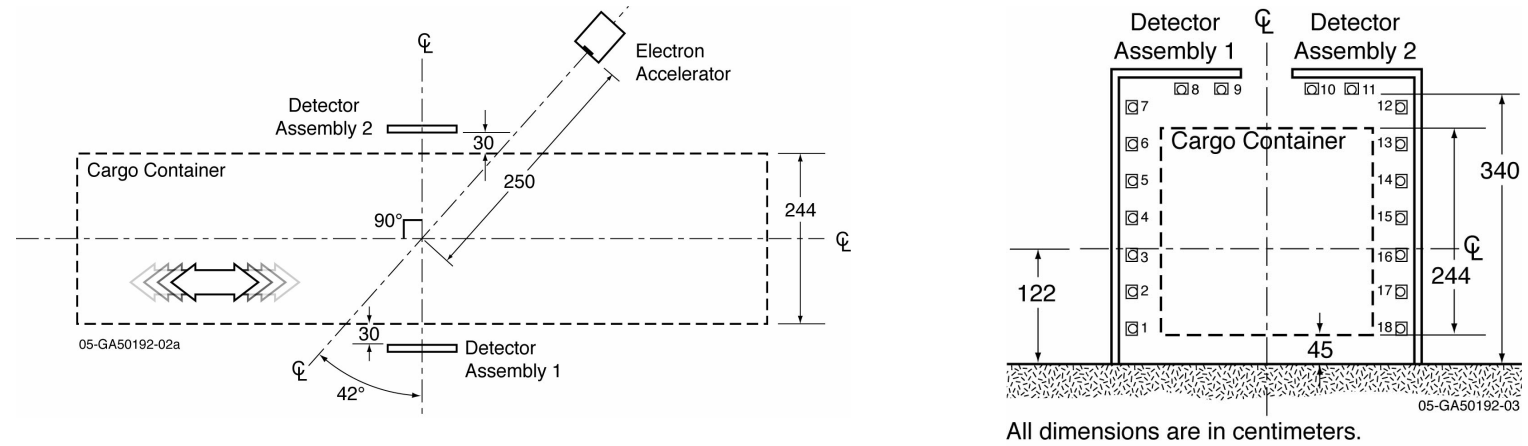

Figure 3. Cargo configuration schematic top view (left) and end view (right). The detector location numbers are shown in the "end view." 


\section{GAMMA-RAY DETECTION CHARACTERIZATION}

In an effort to investigate the possibility of improving/enhancing the PPA gamma-ray detection system, several tests were conducted using a variety of gamma-ray detectors and acquisition schemes. These tests included evaluation of several GM detectors, an initial appraisal of a fast plastic scintillator as a possible improvement over the PPA-utilized GM detectors, and the assessment of an INL-modified High-Purity Germanium detector. An effort was also directed towards overall improvement to the data acquisition software for both gamma-ray and neutron detection. This section of the report highlights the experimental setups, the results from the data collected during those experiments, and the selected improvements implemented for optimal data acquisition.

Currently, GM tubes are being exploited as a means of gamma-ray detection on the PPA system. In the early prototype PPA system, simple counting with the GM detectors was evaluated as a means to enhance the sensitivity to nuclear material within low-Z shielding configurations. Gamma-ray detection provides a method to compensate for reduced neutron signature with low- $Z$ shielding materials and, hence, enhances overall PPA system performance. In the presence of low- $Z$ and highly hydrogenous shielding, neutron detection is extremely difficult due to the rapid thermalization and subsequent neutron absorption which depletes the neutron population. In such shielding configurations, it is possible, however, to utilize cargo-emitted gamma-rays to indicate the presence of nuclear material. The utilization of GM detectors to enhance low-Z shielded nuclear material arises from the excess gammas produced from the delayed effect from fissioning nuclear materials. These excess photons only exist when fissionable materials are present. While the CY05 report presented an initial attempt to deploy gamma-ray detection with GM counters, optimizations were needed before complementary implementation with the PND system could be undertaken. This optimization first required a thorough characterization of the proposed GM tube before a specific model could be recommended. The two primary factors considered in the selection of the GM tube to be deployed on the PPA system were:

1. Operation after the accelerator pulse and dead time.

2. Efficiency of the GM tube.

The first concern is mitigated by the fact that in any detected event, a complete ionization of the gas near the anode is induced. This is independent of the number of photons which cause an ionization event and/or the energy of the incident radiation. In a pulsed photon environment this is extremely important since a large number of photons will interact in the tube during and immediately after the interrogating photon pulse (i.e. flash). This inherent property of GM tubes makes them ideal candidates for use in a pulsed photon environment. In particular, this flash response is independent of the interrogating electron beam energy. In most GM tubes, dead times range from approximately 50 to 200 $\mu$ s and provide count rates on the order of $10^{4}$ counts per second. These are intrinsic characteristics of GM tubes and, to some degree, governed the selection of the tubes that were assessed. The final consideration, tube efficiency, was determined through a combination of researching manufacturer specifications as well as in-depth experimental assessment.

\subsection{Geiger-Müller Detector Evaluations}

GM tubes have novel operational capabilities within a photon flash environment and operational performances were assessed for specific models. 


\subsubsection{GM Detectors In The Flash}

Based on manufacturer specifications, which fit the general requirements of the PPA system, four GM tubes were selected for evaluation. LND Inc. offered the range of GM tubes with the desired specifications and was the sole manufacturer of the GM detectors tested. The models selected were the LND 719, 740, 72511, and 72120. To address the GM operation after the photon flash, the LND 719 and LND 740 GM tubes were evaluated. Tube responses were initially observed on an oscilloscope and acquired after the flash with a multi-channel scalar (MCS). The LND 719 was utilized in the early tests of the PPA system. These tubes have a listed 100-microsecond dead time (160 $\mu$ s as measured with an oscilloscope) which is dictated by the mobility of positive gas ions and the resistor-capacitor (RC) time constant. The LND 740 tube had a $65 \mu$ s dead time. The RC time constant used was based on the manufacturer recommended circuit for each GM tube used. The resulting MCS data for these two detectors during a representative inspection are shown below.

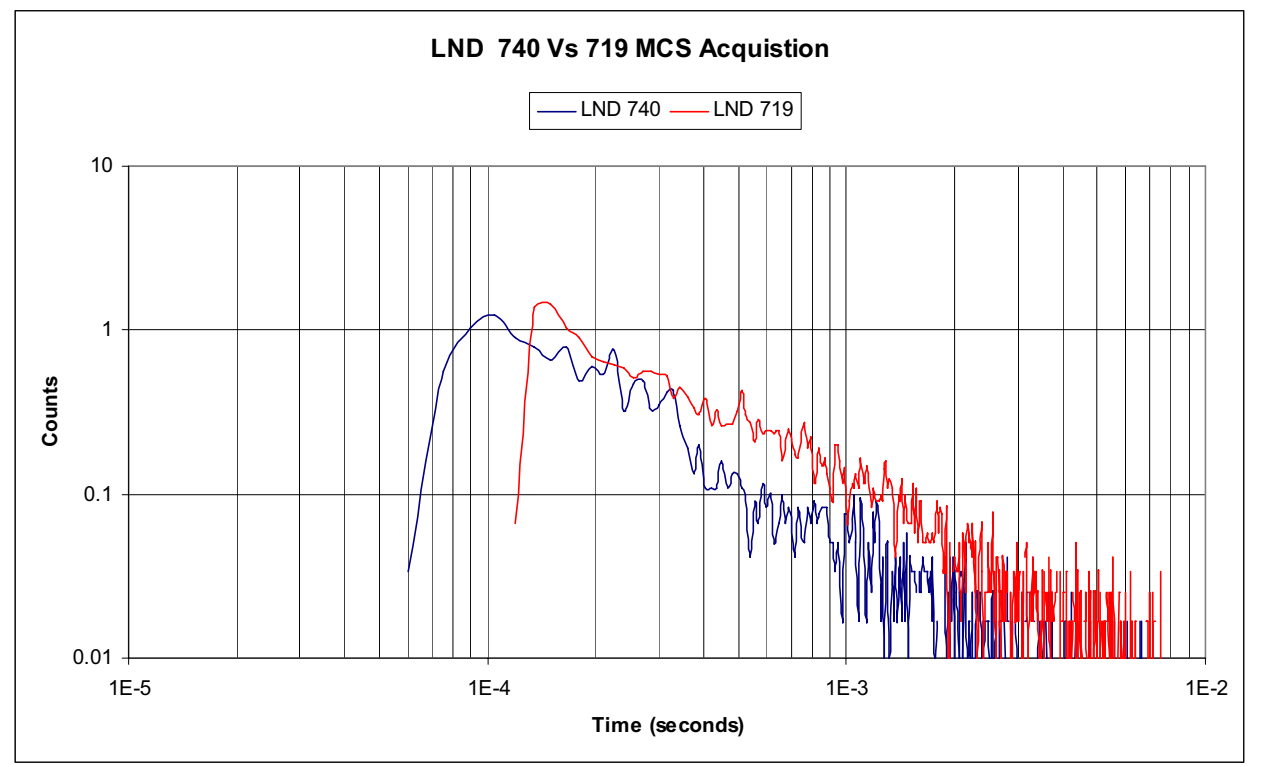

Figure 4. MCS data collected for GM detector models LND 719 and LND 740 during a photon inspection.

As Figure 4 illustrates, although the LND 740 recovered from the flash in $60 \mu$ s, it didn't reach its full efficiency until about $80 \mu \mathrm{s}$. This was faster than the LND 719 which recovers at about $150 \mu \mathrm{s}$. The faster recovery of the LND 740 is an effect of the smaller tube design; however, this design also reduces the efficiency of the detector. Because of the need for increased counting statistics, efficiency becomes a more important factor than recovery at this point of the PPA system development. In the delayed counting region-of-interest (ROI) acquisition window, the 719 recorded approximately five times the number of photon counts when compared to the 740 performance.

\subsubsection{GM Detector Voltage and Dead Time Performance}

To gain a better understanding of GM counting efficiency as a function of input voltage and to verify manufacturer specifications, voltage plateau curves were developed. Details relative to these curves are presented in Appendix A. For gamma-ray detection applications, the operational voltages were $900 \mathrm{~V}$ for the 719, $700 \mathrm{~V}$ for the 740, and $500 \mathrm{~V}$ for both the 72511 and 72120 detectors. Each curve includes the dead time corresponding to its temporal response regardless of its passive or active interrogation operation. 

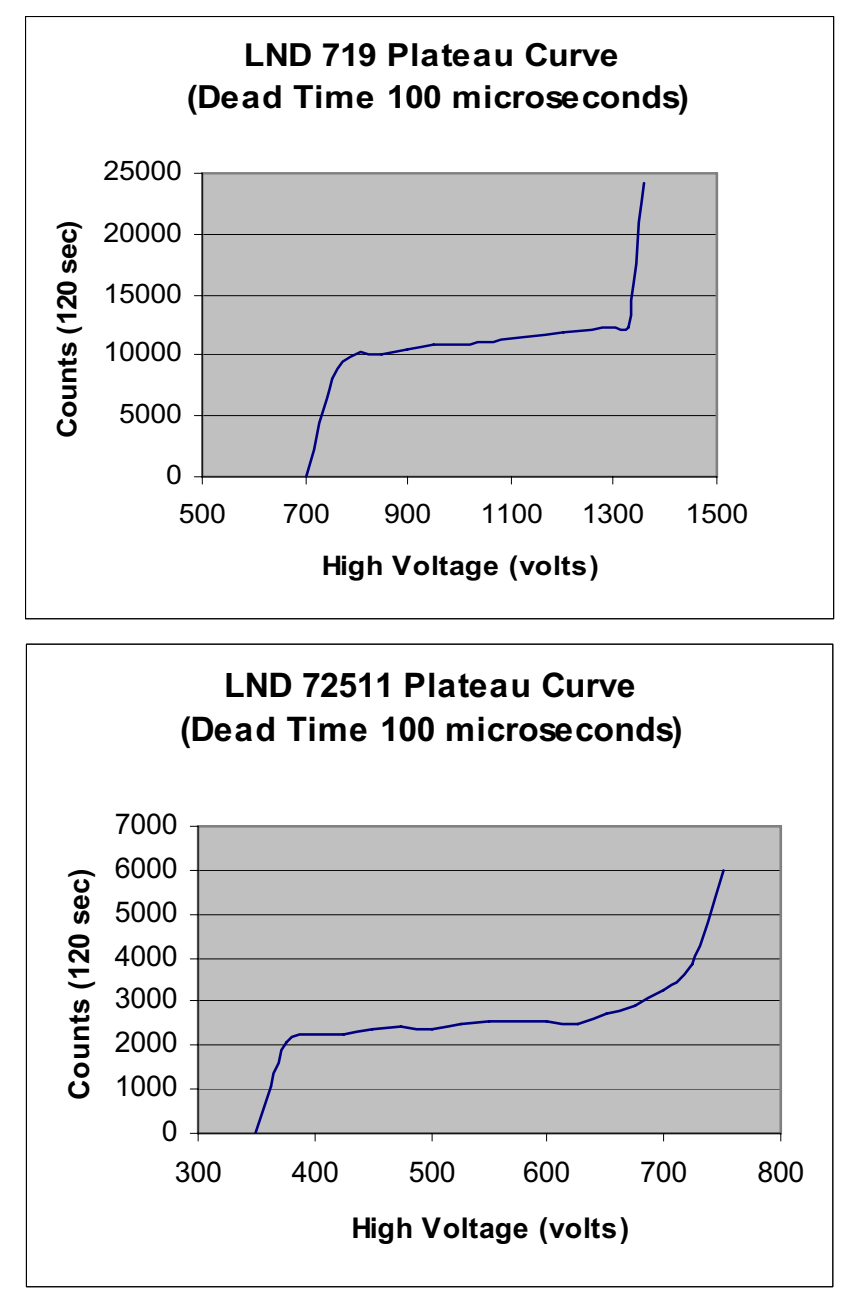
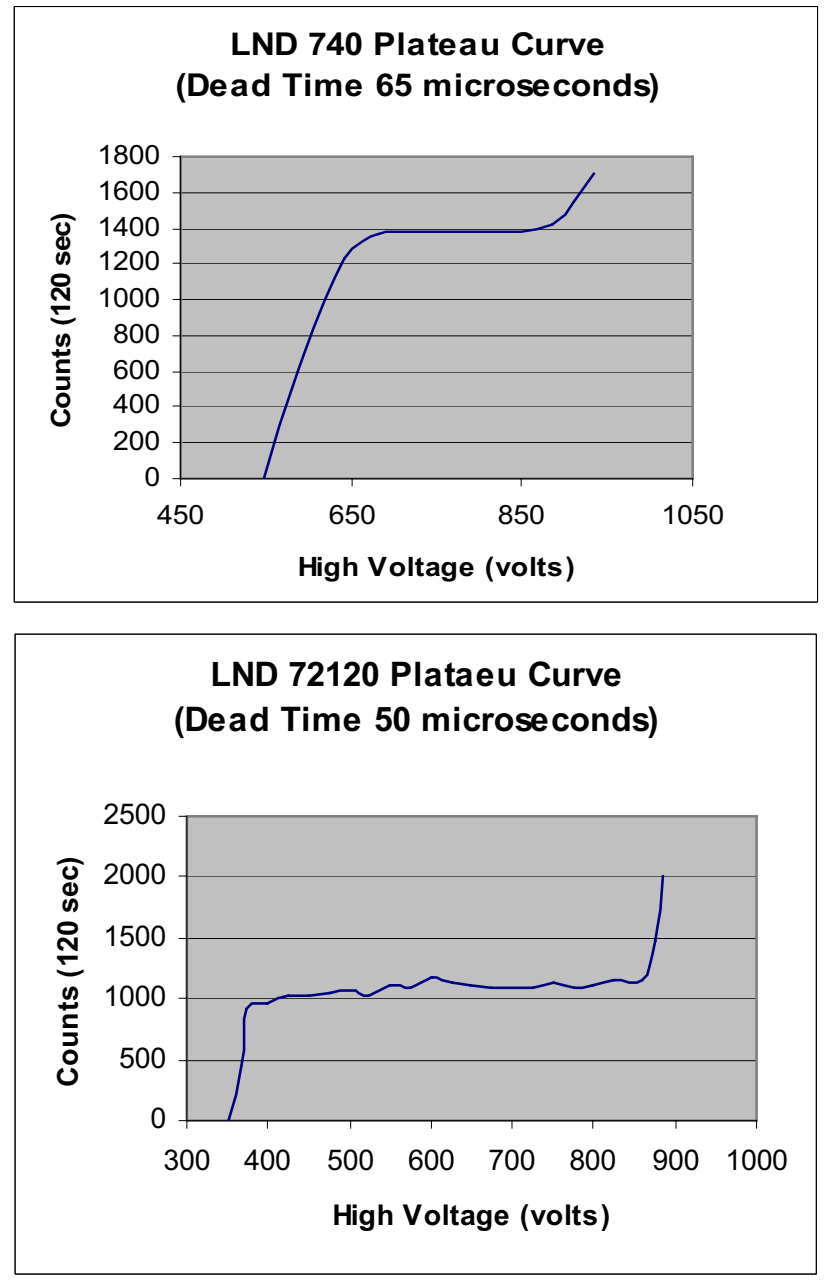

Figure 5. Voltage plateau curves and dead time responses for selected LND Gamma-Ray detectors.

Based on the experience with the LND 719 and 740 detectors, it became apparent that dead time performance alone was not a sufficient metric on which to base the GM tube selection. The second consideration listed above (efficiency) became the primary criteria for detector selection.

Detection efficiency is approximately linear with respect to detector surface area. Given this relationship, it seems that one would simply choose the largest possible detector to increase efficiency. However, response times of the GM tubes are directly proportional to the total effective detection volume. As detector volume is increased, the recovery time decreases. Although the LND 740 could recover 35 $\mu$ s faster than the 719 (see Figure 5), count rates using the 740 were decreased by a factor of eight. Figure 5 also shows that while the 740 and 72120 tubes recover more quickly that the 719 and 72120 , the loss of effective detector area and hence, the loss of effective count rates, leads to the conclusion that the 719 tube is a more appropriate choice for PPA applications at this stage of system development. 


\subsection{Neutron and Gamma Detector Normalization Factors}

A critical component in the optimization of the detection algorithm used for the PPA system data acquisition scheme was found to be the normalization of both the gamma-ray and neutron signals. Normalization of individual detector responses was necessary due to variations in detector-to-detector count rates in the pulsed interrogation environment. The variations in non-normalized individual detector counts were leading to higher than expected false alarm rates. This was due to higher count rates created by individual and summed PND and GM detectors. Variations in identical radiation detectors were attributed to several factors including: individual detector electronics, detector physical location, and slight variations in tube efficiencies. Normalization of all system detectors improved coefficient variations by $385 \%$ in the PNDs and $540 \%$ in the GMs. By normalizing the PPA detection system, the false alarm rates were significantly reduced for interrogations without nuclear material and significant improvements were noted in the detection of nuclear material in low-Z shielding configurations.

\subsubsection{Neutron Detector Normalization}

The Varitron linear accelerator was operated at a nominal $10-\mathrm{MeV}$ with a $125-\mathrm{Hz}$ repetition rate. A total of ten inspection data sets were collected. Each corresponded to a 10-minute inspection time and all data were collected in the delayed region-of-interest window (1.92 to 8 milliseconds) after each interrogation pulse. The Polyethylene Calibration pallet was used as a shield material enclosing a $4.8-\mathrm{kg}$ depleted uranium (DU) target. Counts from all 19 PNDs, plus the four background detectors, were recorded. The top schematic of Figure 6 illustrates the raw counts from these ten acquisitions. Over the course of the ten runs, each detector showed a consistent deviation from the mean counts. The average coefficient of variation of the raw counts was $22.12 \%$. A correction factor for each detector was calculated based on the average deviation from the mean counts. After applying the correction factors, the average coefficient of variation was $5.74 \%$. This resulted in a $385 \%$ reduction of the coefficient of variation and is shown in Figure 6 (center). The applied correction factors, which are based on the coefficients of variation, are plotted in Figure 6 (bottom). 


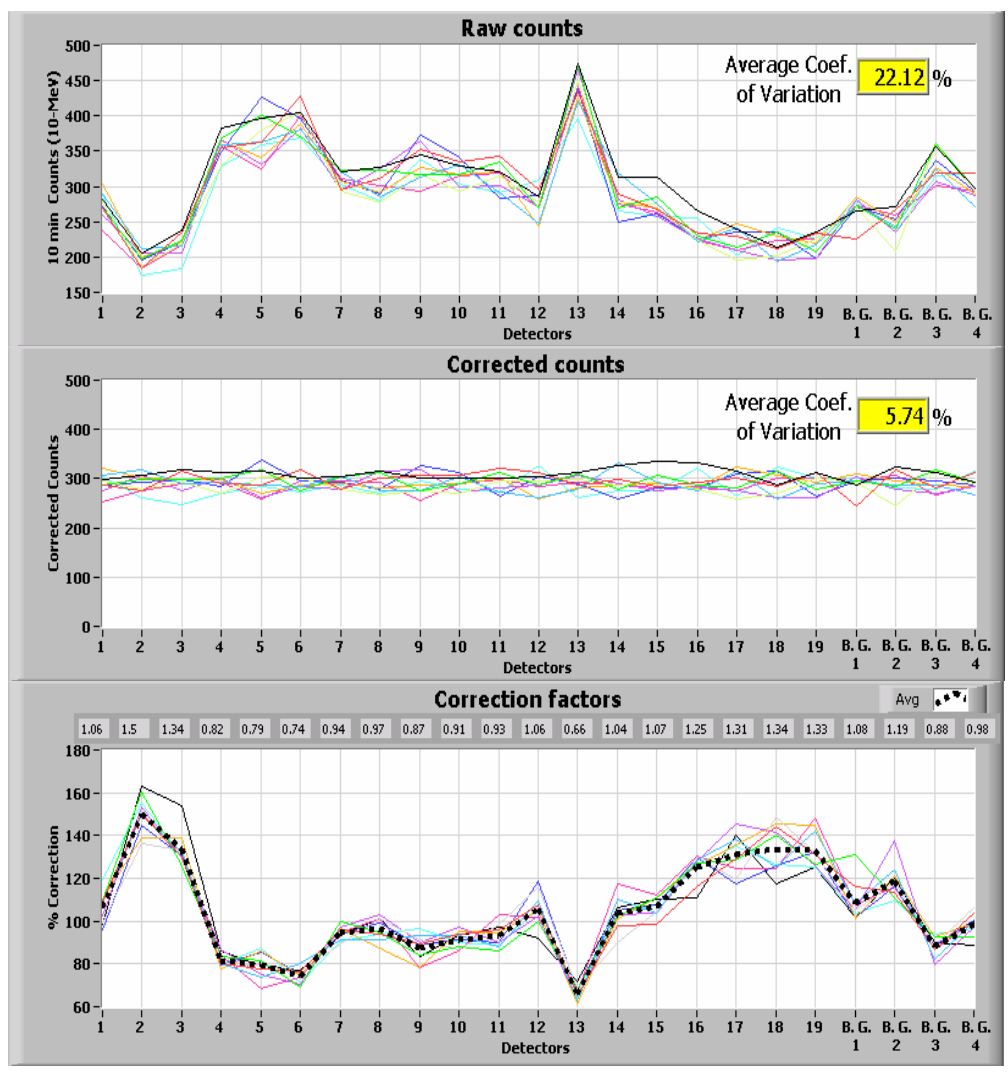

Figure 6. Multiple investigations of raw PND data (top), normalized PND data (center), and PND normalization factors (bottom).

\subsubsection{Gamma-Ray Detector Normalization}

The same procedure used to normalize the PND neutron detectors was applied to the GM detectors. The accelerator was operated at the same frequency and nominal energy and data were acquired in the same delayed time region. Counts from GM detectors \#5-18 were recorded. Detectors \#1-4 were under development at the time of data collection; however, subsequent characterizations on these detectors have been completed. Figure 7 (top) displays the raw counts from the ten repeated interrogations. As in the PND normalization, each detector displayed a consistent deviation from the mean counts. The average coefficient of variation of the raw counts proved to be $9.68 \%$. A correction factor for each detector was calculated based on the average deviation from the mean counts. After applying the correction factors to individual detectors, the average coefficient of variation was reduced to $1.78 \%$. Application of the correction factors resulted in a 540\% reduction of the coefficient of variation. This is shown in Figure 7 (center). The applied correction factors, which are based on the coefficients of variation, are plotted in Figure 7 (bottom). 


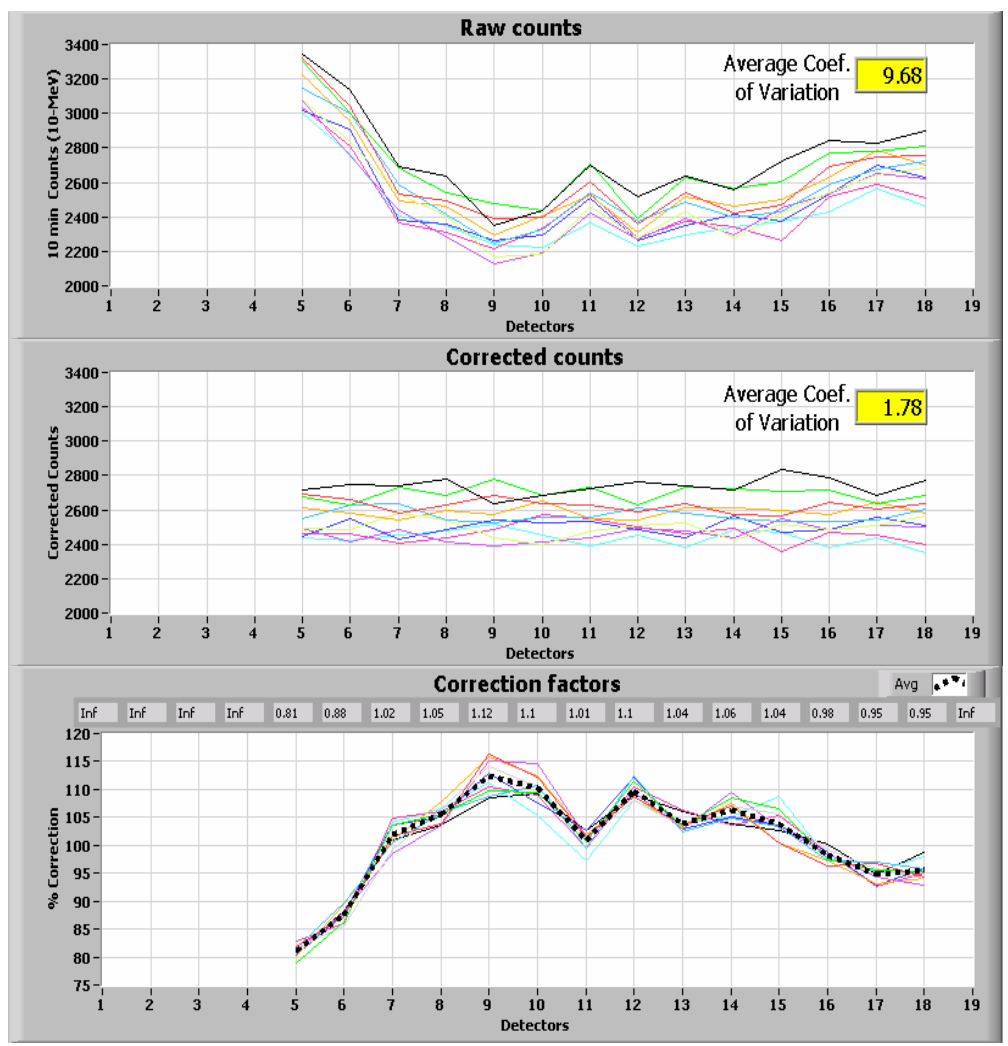

Figure 7. Raw GM data (top), normalized GM data (center), and GM normalization factors (bottom).

\subsubsection{GM Detection Figure-of-Merit Algorithm}

When material is interrogated with photons of sufficient energy, prompt neutrons and gamma-rays are emitted. These prompt neutrons quickly thermalize within a few milliseconds after each interrogating pulse. This thermal neutron environment generates copious additional gamma-rays primarily through neutron capture $[(\mathrm{n}, \gamma)]$ and inelastic scattering $\left[\left(\gamma, \gamma^{\prime}\right)\right]$ reactions leading to a gamma-ray background that could easily mask any induced gamma emissions from a concealed nuclear material. An improved signature method is proposed that attempts to compensate for this actively induced, gamma-ray background. This method utilizes the response of a bare He-3 detector, co-located with the PPA detector arrays, to monitor the thermal neutron environment $\left(\mathrm{n}_{\mathrm{th}}\right)$, and integrates the time-dependent gamma-ray response GM detector $(\gamma)$. This signature method (see Equation 1) develops a time ( $\mathrm{t}$ )-dependent Figureof-Merit (FOM) that is mathematically evaluated after each accelerator pulse $\left(t_{p}=\right.$ time after each pulse corresponding to the start of a selected delayed ROI) and compares it to a predetermined, detection-based, time-dependent signature. 


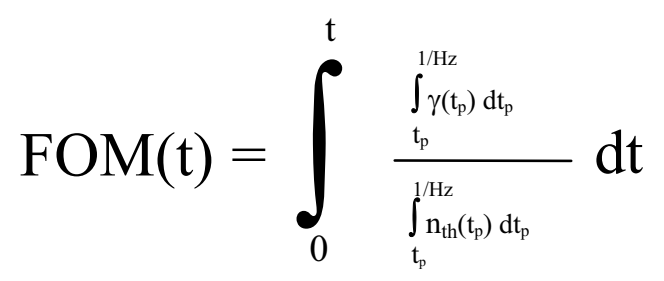

Equation 1. Merit calculation for gamma-based detection algorithm.

This method relies on the fact that gross, time-dependent, thermal neutron capture response, measured by a GM tube per induced thermal neutrons during a pulse inspection, is relatively insensitive to changing cargo loadings. Any additional delayed gamma-ray contribution from a nuclear material, such as prompt gammas from thermal neutron fissions, or gamma-rays from the decay of delayed neutron precursors, will increase the value time-dependent of the FOM. Further improvements in the FOM can be derived by optimizing the gamma-ray ROI.

\subsubsection{Gamma-Ray Signatures versus Selected Regions-of-Interest}

The time-dependent evolution of gamma-ray counts in the delayed counting region depends strongly on the average $\mathrm{Z}$ of the surrounding material and whether there is nuclear material present. If nuclear material is present, the ratio of gamma counts to thermal neutrons (as measured by the bare neutron detector) will deviate from a time-dependent response associated solely with thermalization and capture of neutrons in the inspected object without a nuclear material.

In order to investigate the variation in gamma-ray detection efficiency as a function of the delayed time window, the wood pallet was placed in the beam and data were acquired with and without DU. Three different acquisition windows were used: A) 1.92 milliseconds (ms) $-8 \mathrm{~ms}$, B) $4 \mathrm{~ms}-8 \mathrm{~ms}$, and C) $6 \mathrm{~ms}-$ $8 \mathrm{~ms}$. Gamma counts were summed over the three windows and divided by the thermal neutron counts over the same window as indicated by Equation 1. The FOM ratio of the quantity for $\gamma / n_{\text {th }}$ with DU to $\gamma / n_{\text {th }}$ without DU is given below for each time window based on 120 -second inspections:

Window A (1.92-8 ms): $1.257 \pm 0.058$

Window B (4-8 ms): $1.385 \pm 0.097$

Window C (6-8 ms): $1.389 \pm 0.160$

As the time window is moved further out into the delayed region, the delayed gamma signature with nuclear material present is accentuated. Unfortunately, this puts interrogation limits on the pulsing rate of the photon source, and at longer delayed times, the number of counts decreases resulting in increased measurement uncertainties.

\subsection{Gamma Spectra Utilizing High-Purity Germanium Detectors}

A spectroscopic, gamma-ray detection technology, provided by an INL-modified, High-Purity Germanium (HPGe) detection system, was assessed as a possible complement to the PPA system. The HPGe detector offered the ability to detect short-lived fission fragments generated through photofission. Using a nominal $125-\mathrm{Hz}, 10-\mathrm{MeV}$ inspection operation, the HPGe acquired data between accelerator 
pulses. Although the HPGe detector offers the ability to recover a few microseconds after the pulse, a delay gate was used to acquire data in a 4 to $8 \mathrm{~ms}$ time window (corresponding to the last half of the available time between pulses).

Data was acquired from several experiments with a lead-shielded, HPGe detector placed in a vertical plane located one meter away from the photon source (Varitron converter) and $70 \mathrm{~cm}$ below the beamline. A 1.2-kg DU plate was placed one meter from the photon source and on the beam axis. With no photon interrogation, Figure 8 presents the spectra with and without the DU material. The background was enhanced to include a ${ }^{60} \mathrm{Co}$ calibration response. The ${ }^{243 \mathrm{~m}} \mathrm{~Pa}$ peak from DU at $1.001 \mathrm{MeV}$ was easily distinguishable. Figure 9 shows the corresponding spectra using a nominal $10-\mathrm{MeV}, 125-\mathrm{Hz}$ operation. A major short-lived peak from ${ }^{94} \mathrm{Sr}\left(5.1\right.$-s half-life) and ${ }^{96} \mathrm{Ag}(75.2$-s half-life) at $1.42 \mathrm{MeV}$ is apparent. This combined peak shows a count rate of 1.79 counts per second (cps). When comparing to the DU spectra, an additional 220,400 counts in the 300 second acquisition time, or $734 \mathrm{cps}$ (for energies between 0.5 to $5.2 \mathrm{MeV}$ ) are present when DU is included.

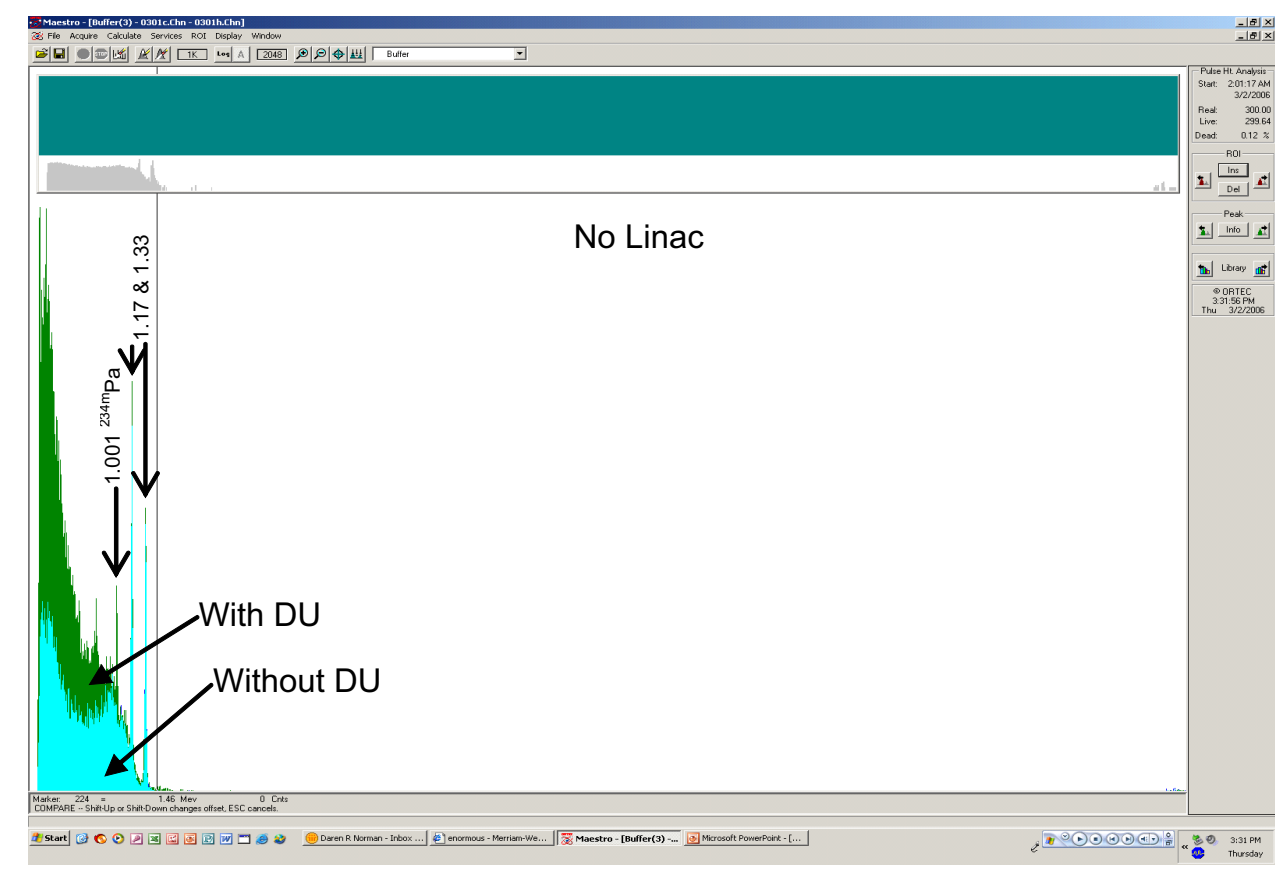

Figure 8. Background without accelerator operations, with and without DU. 


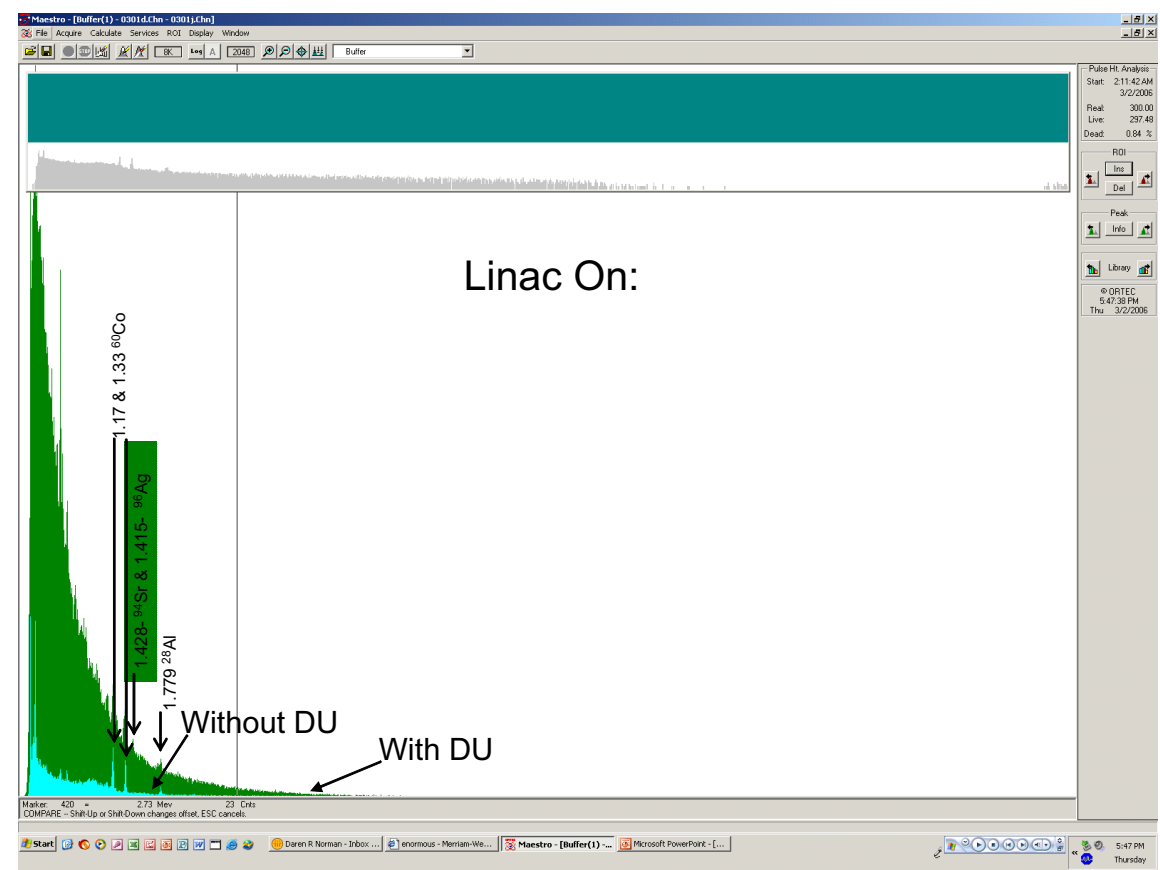

Figure 9. Gamma-ray spectra for a 10-MeV, 125-Hz Linac operation with and without DU.

The HPGe detector offers an excellent tool for system diagnostics. As expected, when nuclear material is present, the HPGe provides identifiable nuclear material-based delayed counts that the GM tube detects. This series of assessments proved that the HPGe detector can not only be used to detect the DU, it also offers energy-dependent data to aid in system development. However, the cost associated with a wholesale substitution of the PPA system's GM detectors with HPGe detectors would be prohibitive; and hence, has not been recommended.

\subsection{Fast Plastic Scintillator Response}

As another possible alternative to gas-filled GM tubes, fast plastic scintillators were investigated as a gamma-ray detection method within the PPA system. The most common organic scintillator is based on a matrix of polyvinyl toluene (PVT). It is dissolved in a solvent and subsequently polymerized forming the equivalent of a solid solution. These scintillators are relatively inexpensive, can be shaped to any form necessary, and provide good sensitivity for $0.1-\mathrm{MeV}$ to $5 \mathrm{MeV}$ photons. The primary advantages of using plastic scintillators are their fast response time ( $2.5 \mathrm{~ns})$, photon sensitivity (100-500 times better than GM), and their ability to use energy information to discriminate spurious signals due to background. The associated electronics with scintillators are also quite simple since the light output and electron conversion are about three orders-of-magnitude greater than the signal from a GM tube.

\subsubsection{Fast Plastic Experimental Configuration}

To investigate these advantages, a simple test was conducted using a BC-408 (EJ-200) scintillator (6-cm diameter, 6-cm length) coupled to a Photonis photomultiplier tube biased at $-1280 \mathrm{~V}$. The detector was surrounded by one standard $2.54-\mathrm{cm}$ thick lead brick thickness to reduce background and to collimate the field of view directly toward the center of the Polyethylene Calibration pallet. Because of spatial restrictions, the detector assembly was positioned orthogonally to the accelerator beamline at a distance of $122 \mathrm{~cm}$ from the center of the pallet, which replicates the spacing to GM detectors \#3 and \#16 (refer to 
Figure 3). Figures 10 and 11 illustrate the experimental setup. Two specific responses were investigated using the scintillator: (1) recovery after the flash (2) delayed photon counting.

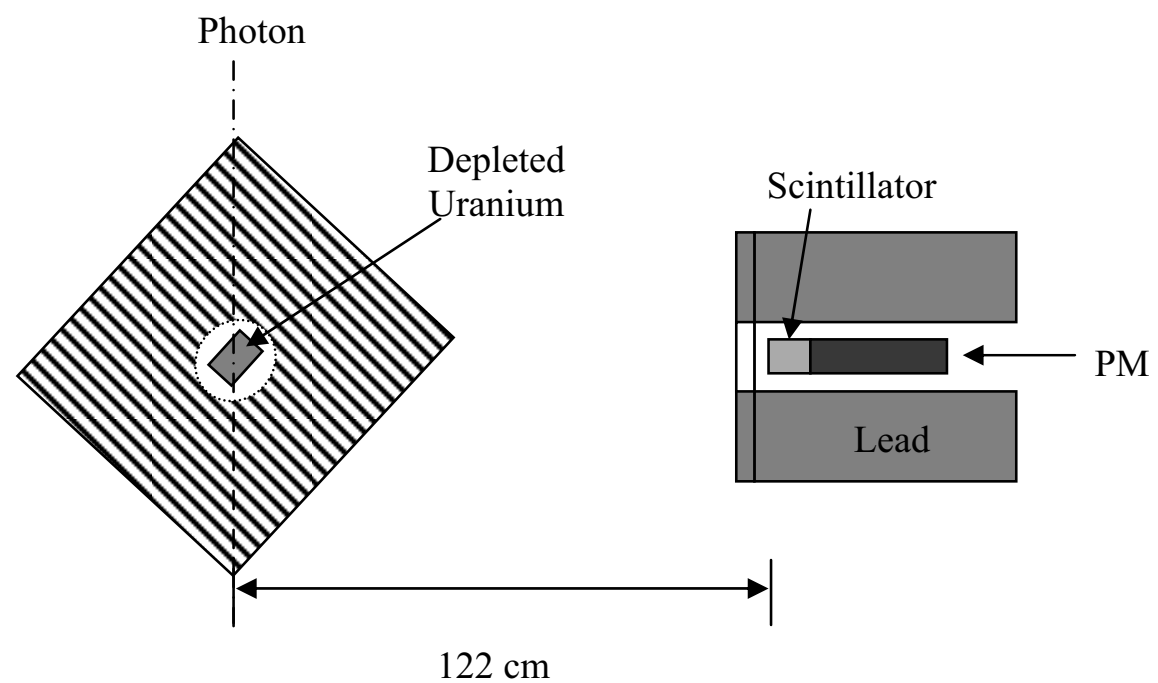

Figure 10. Plan-view illustration of experimental setup. Note that the lead shield completely surrounds the entire detector with the exception of the front and rear faces.
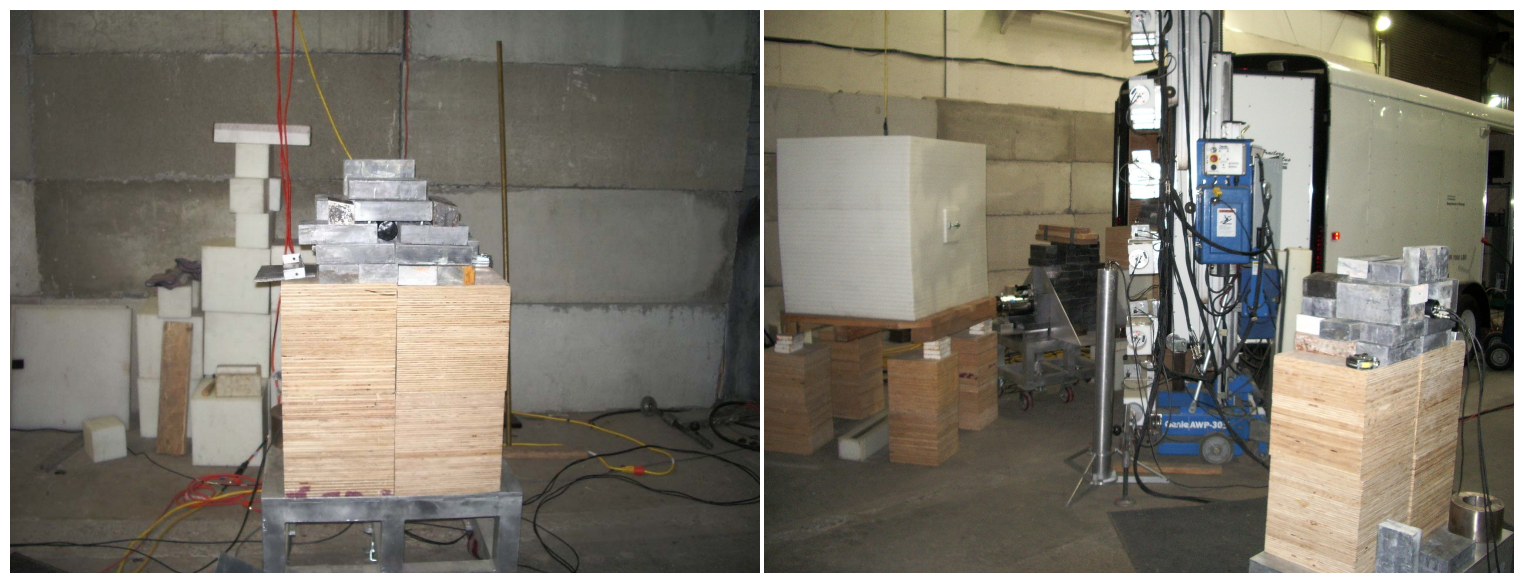

Figure 11. Photographs of experimental setup illustrating relative positions of the polyethylene pallet and shielded detector assembly (left side view is from Polyethylene Calibration Pallet).

To ensure the scintillator was responding only to photons, energy discrimination was required. This was accomplished by calibrating photon energy response with a ${ }^{60} \mathrm{Co}$ source. The pulse-height signal corresponding to the Compton-edge $(\sim 1 \mathrm{MeV})$ was measured with an oscilloscope. Using this pulse height as a reference, signals from the detector/PMT were passed through a $40-\mathrm{MHz}$, constant fraction discriminator (CFD) which was set with the lower level discriminator at $0.5 \mathrm{MeV}$. It is important to realize that the signal output from photons in $\mathrm{BC}-408$ is nearly 2.5 times that of an equivalent energy neutron. Therefore, by setting the discriminator at $0.5 \mathrm{MeV}$ (electron energy equivalent), neutrons below $1.25 \mathrm{MeV}$ were discriminated. This is useful in looking at signals a few milliseconds after the photon flash. In this time regime, nearly all fast neutrons have thermalized and are well below $1.25 \mathrm{MeV}$. 


\subsubsection{Recovery after the flash}

To assess response after the flash, the MCS was modified so that the dwell time on each acquisition channel was reduced to $100 \mathrm{~ns}$. Counting response from the GM (LND 719) detectors and scintillator was investigated. As expected, the scintillator was capable of counting immediately after the flash. As illustrated in Figure 12, the scintillator was able to count within the first $100 \mathrm{~ns}$ after the flash. It should be noted that the GM detectors did not begin counting until a significant time after the flash. While the GM response was sufficient in the delayed time region $(>2 \mathrm{~ms})$, the fast response of the scintillator opens the possibility of pushing the counting region closer to the flash and perhaps even into the prompt regime during the flash.

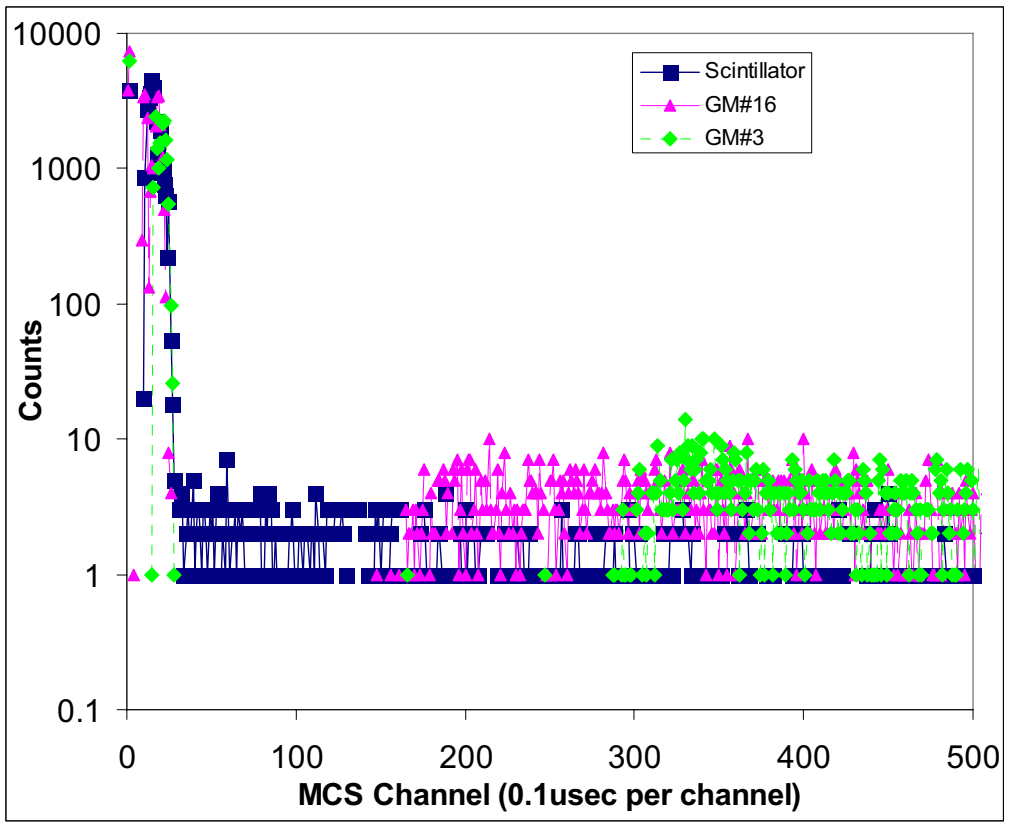

Figure 12. Time response of scintillator and GM detectors immediately after the flash.

\subsubsection{Nuclear Material Detection}

With the lower level photon discriminator set to $0.5 \mathrm{MeV}$, tests were conducted using the setup shown in Figure 11. Several $10-\mathrm{MeV}$ inspections were conducted with and without depleted uranium present in the Polyethylene Calibration Pallet. Using the FOM equation, photon counts were summed over varying time intervals and divided by the bare detector (thermal neutron) counts over the same interval. Figure 13 shows the results of the FOMs with and without DU for GM \#3, GM \#16, and the scintillator. The respective GM detectors were chosen because of their location on the detector assembly. Along with the scintillator, these detectors were located at the centerline height of the DU in the pallet. 


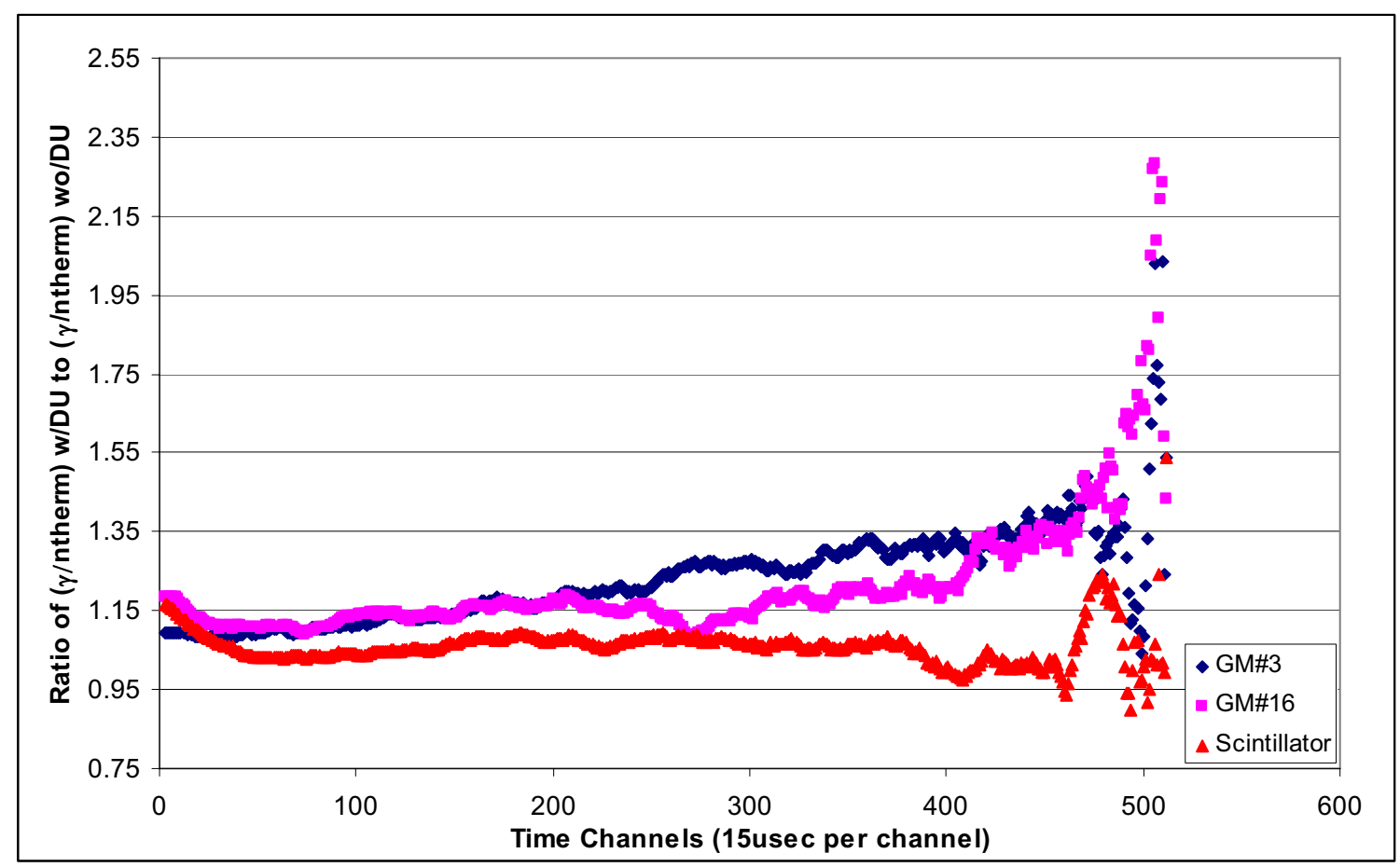

Figure 13. Ratio of the FOM for the polyethylene pallet (DU/no DU)

It is apparent from Figure 13 that the GM detectors actually perform better in the delayed regionof- interest. However, at the earliest times $(<300 \mu \mathrm{s})$, or 20 channels, the scintillator performs sufficiently well to detect DU. At latter times (within the delayed acquisition region), the scintillator performance could be enhanced by further lowering the lower level discriminator to approximately $0.1 \mathrm{MeV}$. At this level, the neutron discrimination would still be effective, while the sensitivity to the low energy photons would be enhanced.

Based on the results of the experiments, it was determined that the additional benefit from the speed and sensitivity of the scintillator did not warrant a wholesale substitution in the PPA system at this time. The recovery time of the scintillator is certainly fast enough to move the count window closer to the flash. In fact, scintillators will likely be the primary detector utilized in a follow-on investigation into prompt emission studies. It is possible, however, that larger scintillators that retain their ability to recover after the photon flash may prove in the future to be a viable alternative to the GM detectors. From an economic standpoint, however, it remains to be seen if scintillators of sufficient size and speed can match the low cost advantage of the GM tube. 


\section{OFF-AXIS SENSITIVITY CHARACTERIZATION}

The purpose of this study was to determine the off-axis detection sensitivity of the PPA system during the scanning of a cargo container. For the deployment of any PPA system, it is critical to know how the detection sensitivity varies in relation to the off-axis location of nuclear material.

\subsection{Numerical Simulations and Experimental Benchmarks}

A computer model was first developed to simulate the experimental setup by calculating both photon dose rates $(\mathrm{R} / \mathrm{min})$ and total induced fissions in a 1.2-kg DU billet. The two off-axis experiments described below were designed to provide a benchmark for the MCNPX code ${ }^{16}$ predictions and to provide a valuable and unique opportunity to further utilize and validate the MCNPX code.

\subsubsection{Experimental Setup for Benchmark Testing}

The experimental setup consisted of the following parameters. A $~ 30$-degree (full angle), tungsten collimator was mounted on the beam output and the Varitron was operated at $40 \mathrm{~Hz}$ with a nominal 10$\mathrm{MeV}$ energy. The $1-\mu \mathrm{A}$ average beam current was monitored using a Faraday cup throughout the study. Measurements were made at $2.5 \mathrm{~m}$ and $4.9 \mathrm{~m}$ from the photon source. A multi-detector test assessment configuration was assembled (shown in Figure 14) that included a vertically-positioned PND (with its ${ }^{3} \mathrm{He}$ tube centered on beam axis), a 1.2-kg DU billet measuring $2.54 \mathrm{~cm}$ in diameter and $13.97 \mathrm{~cm}$ in length positioned directly in the center of the PND's ${ }^{3} \mathrm{He}$ tube, and a GM tube (LND 719) centered on beam axis between an ion chamber (dose monitor) and the PND. The experiment is illustrated in Figures 14 and 15. The detector/DU configuration was translated in 30.48-cm increments perpendicular to the center line of the beam for each test at 2.5 and $4.9-\mathrm{m}$ standoff distances.

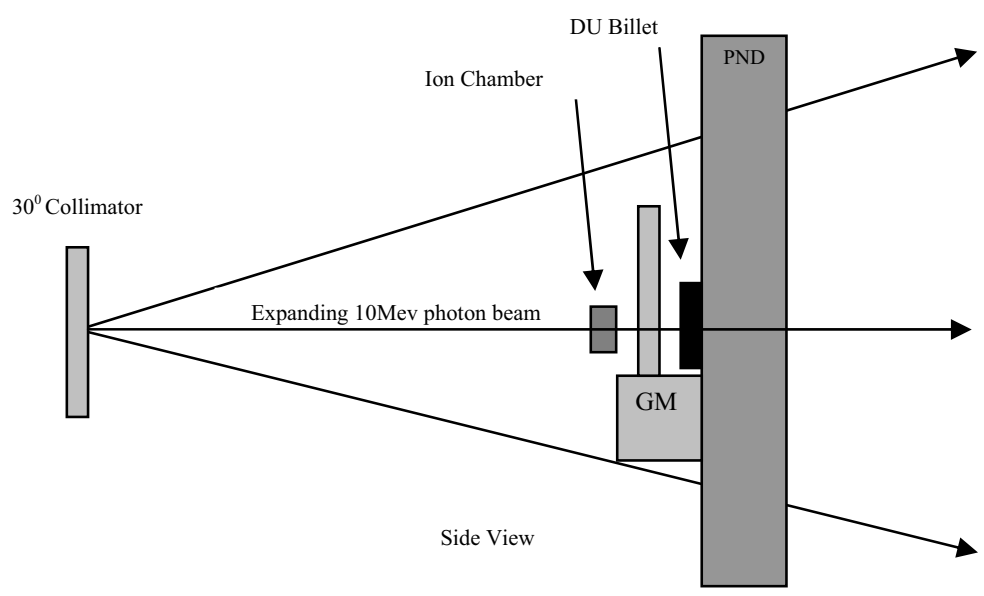

Figure 14. Illustration of beam off-axis experiment geometry. 


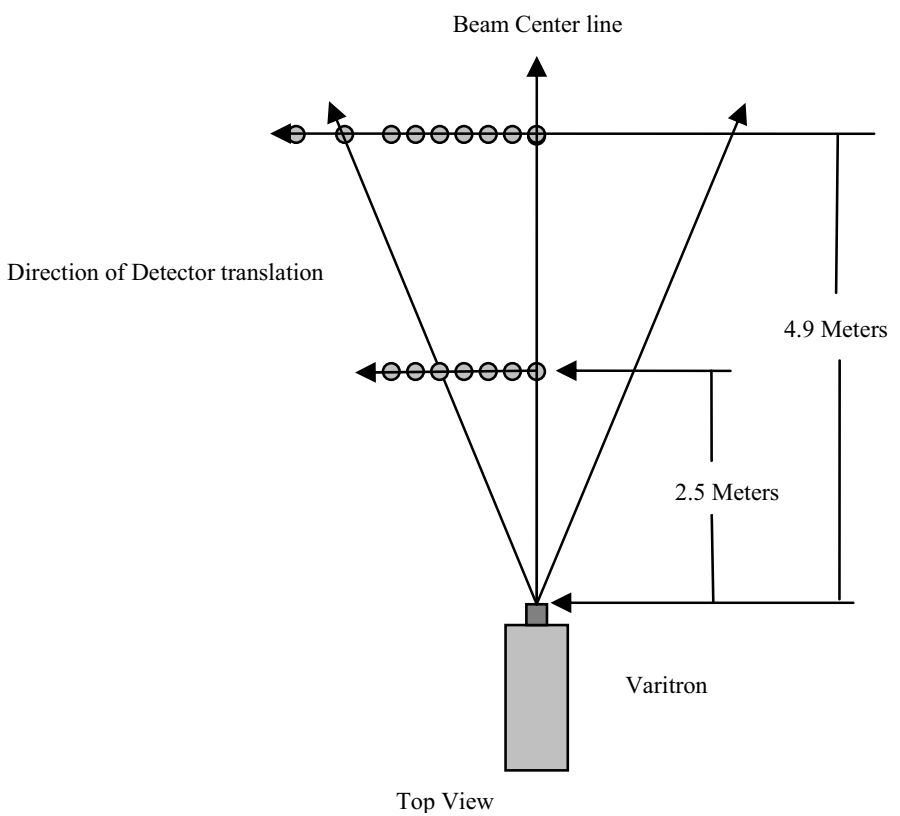

Figure 15. Plan view off-axis experiment geometry.

\subsubsection{Benchmarking}

MCNPX numerical models simulated the accelerator system by means of a normally incident, mono-energetic surface current source of $10-\mathrm{MeV}$ electrons starting on the surface of the electron/photon converter. The converter was the INL Varitron electron-to-photon converter. The electrons were assumed to start uniformly on the converter surface and inside a $0.5-\mathrm{cm}$ diameter circle centered about the beam centerline. The tungsten collimator was initially modeled with a 40-degree full-angle cone aperture. Later measurements showed that the full-angle is actually $\sim 30$-degrees, but this difference should not affect the calculations relative to the subtended angles for the off-axis measurement data.

The 1.2-kg DU billet was modeled. This cylinder was located at $2.5 \mathrm{~m}$ and $4.9 \mathrm{~m}$ along the beamline axis from the converter and systematically moved perpendicularly off-axis to match the experimental measurement locations. The beamline axis, aligned with the center of the converter and DU billet, was measured and modeled to be $135.5 \mathrm{~cm}$ above the floor. Photon dose rates $(\mathrm{R} / \mathrm{min})$ were calculated in front of the DU cylinder at each off-axis measurement location. Figures 16 and 17 compare the calculated photon dose rates to the measured photon dose rates. Data showed acceptable agreement and demonstrated the validity of the MCNPX computer code and models; however, further investigation is needed to fully understand the observed differences in the total photon responses. 


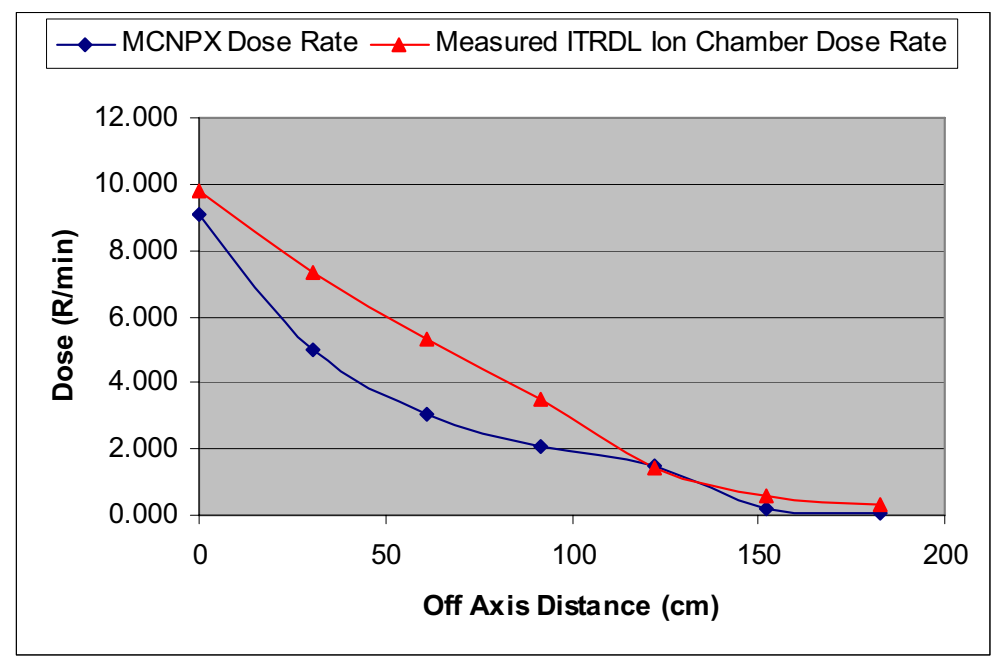

Figure 16. Photon dose rate as a function of off-axis distance for the DU billet at 2.5-m.

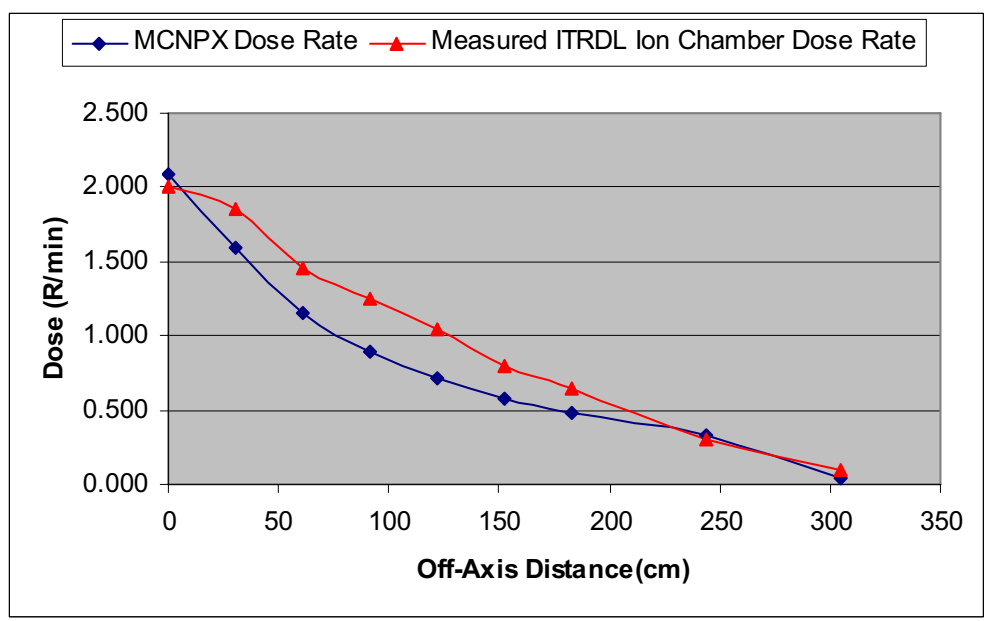

Figure 17. Photon dose rate as a function of off-axis distance for the DU billet at 4.9-m.

In addition, DU delayed neutron counts were measured concurrently with the off-axis photon dose rates. The measured off-axis delayed neutron counts at the two selected beamline positions (i.e., maximum yields) were normalized to the on-axis data. The numerical simulations, predicting the corresponding PND delayed neutron counting responses for each off-axis position, were similarly normalized. These normalized data are shown in Figures 18 and 19. Comparison of the measured and calculated data showed excellent agreement. This demonstrates the validity of the MCNPX computer code to predict high energy photon production and photonuclear interactions. 


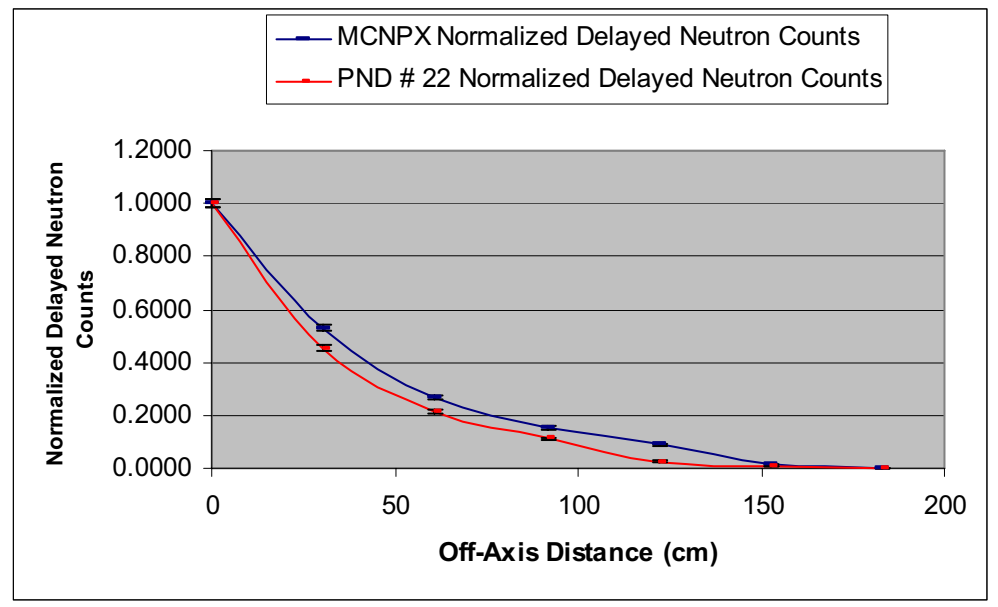

Figure 18. Normalized delayed neutron counts versus perpendicular off-axis distance of the DU billet at a 2.5-meter photon converter-to-DU distance.

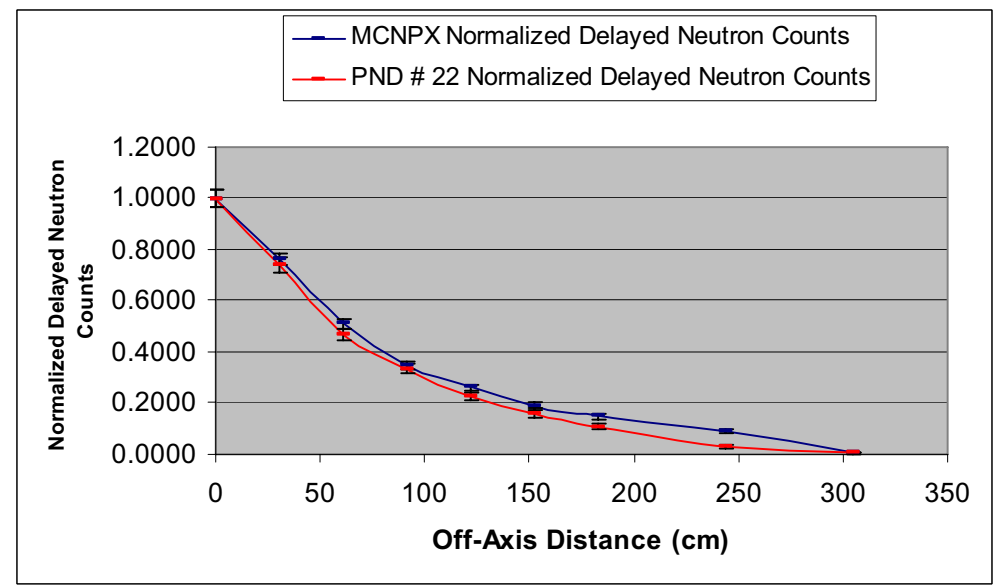

Figure 19. Normalized delayed neutron counts versus perpendicular off-axis distance of the DU billet at a 4.9-meter photon converter-to-DU distance.

Although it will be dependent on the actual shielding material, Figure 20 indicates that a maximum off-axis distance of 27 to $31 \mathrm{~cm}$ (wood) and 32 to $38 \mathrm{~cm}$ (iron) required for detection of $4.8 \mathrm{~kg}$ of DU concealed in a pallet configuration. This trend is consistent with the shielding properties of low- and high$\mathrm{Z}$ materials. Highly hydrogenous shielding materials can be expected to limit the off-axis sensitivity to $\leq$ $30 \mathrm{~cm}$ while higher atomic number shielding should allow nuclear material detection at larger off-axis distances. 
Detection vs. Off-Axis Distance

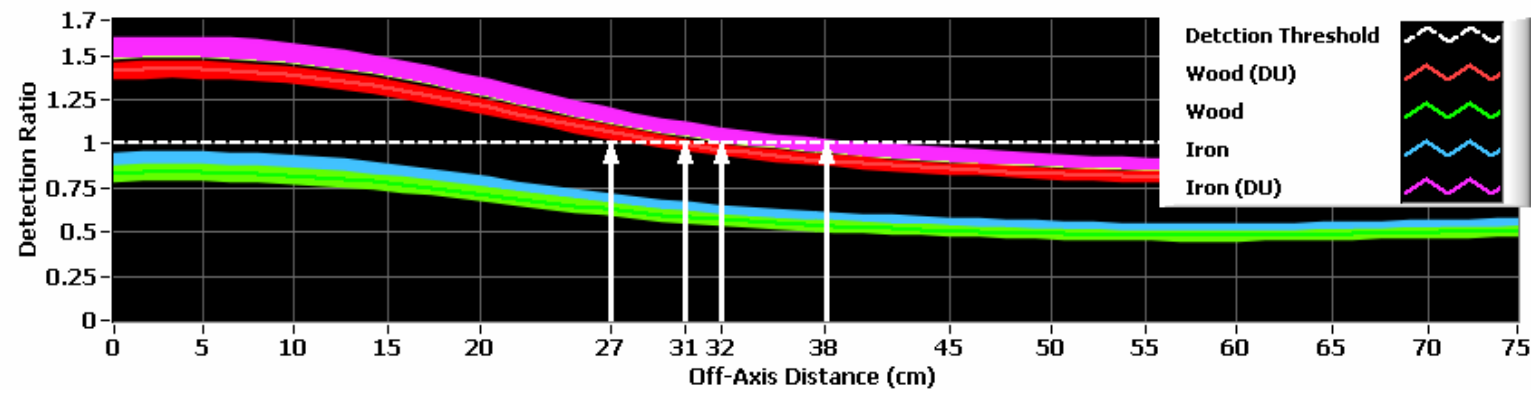

Figure 20. Convolution of experimental pallet data with off-axis MCNPX simulations. 


\section{CONCLUSIONS AND RECOMMENDATIONS}

A prototype inspection system for nuclear material detection has been developed and characterized by INL. The major radiation detection components of the prototype PPA system were built in CY04 and integrated into an inspection system in CY05. Subsequent experiments and characterizations of the PPA detection system have resulted in additional recommendations to further enhance the technology.

With respect to the gamma-ray detection system, different GM detectors, a fast plastic scintillator, and a High Purity Germanium detector (HPGe) were assessed in a pulsed photon inspection environment. The GM (LND 719) detector was shown to have the best overall performance in the pulsed photon environment. Beyond the technical performance of this detector, its low cost and availability make it a logical choice for a field deployable system.

Nuclear material detection was further enhanced by data normalization procedures applied to both the PND and GM detectors. Normalization of all system detectors improved coefficient variations by $385 \%$ in the PNDs and 540\% in the GMs. False alarms have been significantly reduced and improvements were noted in the detection of nuclear material in low-Z shielding configurations. Variation in the counting window within the delayed acquisition region illustrated that the gamma-ray detection signature could be enhanced by looking at a time from 4-8 $\mathrm{ms}$ (corresponding to a $125-\mathrm{Hz}$ Linac repetition rate) rather than the normal 2 to $8 \mathrm{~ms}$ window.

It is recommended that the PPA technology utilizing a GM counting system based on the LND 719 detector be the basis for any future demonstrations. However, a comparably cost-effective gamma-ray detection system that has a better sensitivity and response time than the LND 719 detector may also be suited for this application.

In terms of off-axis detection sensitivity, simulation and benchmarking experiments have indicated that the PPA system can successfully detect nuclear material in typical cargo container inspection configurations even when the material is concealed at a distance of approximately $30 \mathrm{~cm}$ from the beam centerline. It is important to note that the exact detection sensitivity to off-axis interrogations will be dependent on the shielding material. High- $Z$ shielding will allow detection at larger off-axis distances than low-Z materials.

Future demonstrations of this prototype PPA technology will provide the basis for the design of an operational inspection system matched to specified inspection requirements (i.e. CONOPS). 


\section{REFERENCES}

1. J. L. Jones, et al., "Proof-of-Concept Assessment of a Photofission-Based Interrogation System for the Detection of Shielded Nuclear Material", INEEL/EXT-2000-01523, November 2000.

2. D. P. Wells, et al., "CCabinet-safe' study of 1-8 MeV Electron Accelerators," Nuclear Instruments and Methods in Physics Research A, 463, 2001, pp. 118-221.

3. J. L. Jones, et al, "Detection of Pulsed, Bremsstrahlung-induced, Prompt Neutron Capture Gamma-rays with a HPGe Detector," SPIE International Conference: Neutrons in Research and Industry Proceedings, 2867, June 1996, pp. 202-205.

4. J. L. Jones, et al., "Material Identification Technology (MIT) Concept Technical Feasibility Study", WINCO-1147, September 1993.

5. J. L. Jones, et al., "Remote Inspections of Cargo Containers for Nuclear Materials: An Initial Experimental and Numerical Assessment", INEEL/EXT-03-00363, March 2003.

6. J. L. Jones, et al., "Pulsed Photoneutron Interrogation: The GNT Demonstration System", WINCO1225, INL Report, October 1994.

7. J. L. Jones, et al., "Photofission-based, Nuclear Material Detection: Technology Demonstration", INEEL/EXT-02-01406, December 2002.

8. J. L. Jones, et al., "Pulsed Photonuclear Assessment (PPA) Technique: CY04 Year-end Progress Report", INEEL/EXT-05-02583, February 2005.

9. M. T. Kinlaw and A.W. Hunt, "Time Dependence of Delayed Neutron Emission for Fissionable Isotopes Identification”, Applied Physics Letters, Vol. 86, 254104, 2005.

10. D. R. Norman, et al., "Inspection Applications with Higher Electron Beam Energies," Nuclear Instruments and Methods in Physics Research B, Vol. 241, No. 1-4, December 2005, pp. 787-792.

11. D. R. Norman, et al., "Active Nuclear Material Detection and Imaging," 2005 IEEE Nuclear Science Symposium Conference, Puerto Rico, November 2005, Record N20-2.

12. J. L. Jones, et al., "Photonuclear-based, Nuclear Material Detection System for Cargo Containers" Nuclear Instruments and Methods in Physics Research B, 241, 2005, pp. 770-776.

13. J. L. Jones, et al., "Prototypical Integrated Pulsed Photonuclear Assessment System for Nuclear Material Detection," 2005 DHS Conference on Working Together with Industry: Research and Development Partnerships in Homeland Security, Boston, Massachusetts, April 27-28, 2005.

14. J. L. Jones, et al., "Detection of Shielded Nuclear Material in a Cargo Container", ANS Accelerator Applications '05, Venice, Italy, August 2005.

15. J. L. Jones, et al., "Pulsed Photonuclear Assessment (PPA) Technique: CY05 Project Summary Report", INL/EXT-05-01020, December 2005.

16. J. S. Hendricks, et al., MCNPX, Version 2.5e, LA-UR-04-0569, February 2004. 
Appendix A

Geiger-Müller (GM) Experimental Descriptions 


\section{Appendix A}

\section{Geiger-Müller Experimental Descriptions}

The GM tubes were each mounted in an INL provided electronics enclosure that contained the pulse shaping amplifier/buffer and programmable high voltage power supply. The proper signal coupling circuit was used for each detector, as specified by LND Incorporated's Nuclear Radiation Detectors home page located at http://www.Indinc.com/home.htm. GM tube specifications can also be accessed at that link. The ${ }^{60}$ Co source was located approximately $5 \mathrm{~cm}$ from the GM tube, and at the center of the tube active area. Preamplifier/buffer and high voltage module low voltage DC power was provided by an Ortec M4003 NIM module and a Canberra M1000 portable NIM power supply. The buffer provides a TTL level, for each detector pulse, to the Canberra timer/counter input, and count time was set for 180 seconds. The high voltage, as measured at the GM tube anode, was set below the specified minimum for each GM tube, and the voltage for zero counts was established. The high voltage was incrementally increased by 25 or 50 volts depending on the GM model (see plateau plots). At each voltage setting, a 180 -second count was completed. The high voltage was measured using a Tektronix TX3 multimeter and Fluke $80 \mathrm{~K}-6$ high voltage probe. 
Appendix B

Neutron Detector Normalization 


\section{Appendix B}

\section{Neutron Detector Normalization}

The PND correction factors previously calculated in ten-minute runs were applied to the acquisition system. One-minute acquisition data were collected using the latest version of the National Instruments Labview graphical user interface (GUI) to test the correction factors. The accelerator was operated at $10 \mathrm{MeV}$ with the Polyethylene Calibration Pallet in the photon beam, center position. Windowing was the same as the calibration data set. The coefficient of variation was larger overall which can be attributed to counting statistics due to reduced acquisition times ( 1 minute) and therefore the total neutrons counted were decreased. Applying the correction factors resulted in a $160 \%$ reduction of the coefficient of variation (See Figure B-1). It should be noted that the test data utilized was collected at an earlier date and the correction factor was applied in post processing. This implies that all stored data (before normalization was implemented) may be normalized using a standard correction factor.

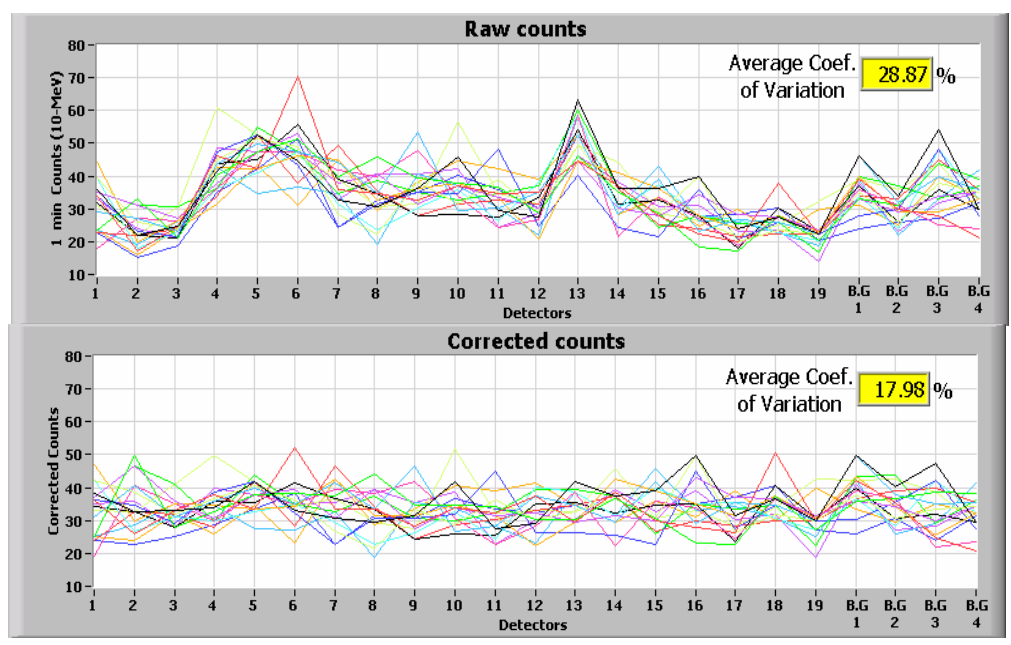

Figure B-1. PND test data (top) with calculated correction counts (bottom).

In addition, four ten-minute runs were acquired with the accelerator OFF. Figure B-2 illustrates a comparison of the PND detector counts with and without an accelerator operating. The average count rate was lower with the accelerator OFF ( 0.301 counts/sec vs. 0.485 counts $/ \mathrm{sec})$ but, more interestingly, the detector trends are quite different indicating that the detectors are influenced by the operation of the accelerator. 


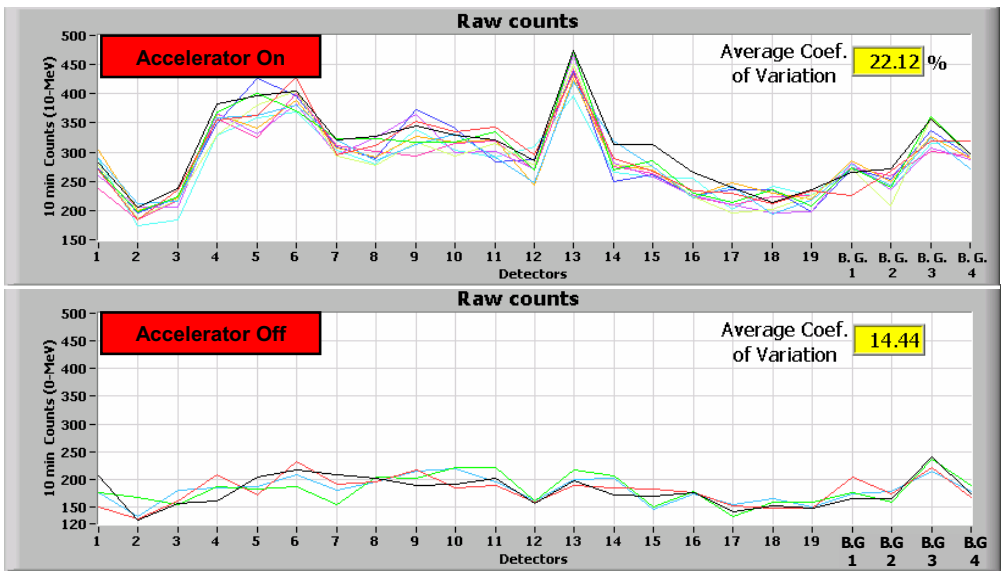

Figure B-2. Accelerator effects on corrected PND responses with accelerator ON (top) and OFF (bottom). 
Appendix C

Gamma-Ray Detector Normalization 


\section{Appendix C}

\section{Gamma-Ray Detector Normalization}

The gamma correction factors previously calculated in ten minute runs were applied to the acquisition system. Data was collected to test the correction factors. It was not possible to correct detectors \#1, \#2, \#3, and \# 4 because they were not available when the correction factors were calculated. Also note that detector \#3 and \#18 were not available in the test runs. Applying the correction factors resulted in a $400 \%$ reduction of the coefficient of variation. Although the coefficient of variation is larger overall because the test data was collected over one-minute runs, the correction factors were still adequate for normalizing the detector responses as can be seen in Figure C-1 (center). It should be noted that the test data used were collected at an earlier date and the correction factor was applied in post processing. The accelerator was operated at $10 \mathrm{MeV}$ with the Polyethylene Calibration Pallet in the photon beam, center position. Windowing was the same as the calibration data set. The spatial ordering of the detectors is the same order as the plot. Note the concave shape of the detector responses in Figure C-1 (top). This is similar to what would be expected of an isotropic point gamma-ray source that is not equidistant to all detectors. If this is the case, it indicates that the variations are not only dependent on detector-to-detector variations (like the neutron detectors) but also on position. Figure $\mathrm{C}-1$ (bottom) shows the raw gamma counts with the accelerator turned OFF. This data set supports the position-dependence theory since the concave form in Figure C-1(top) is not present.

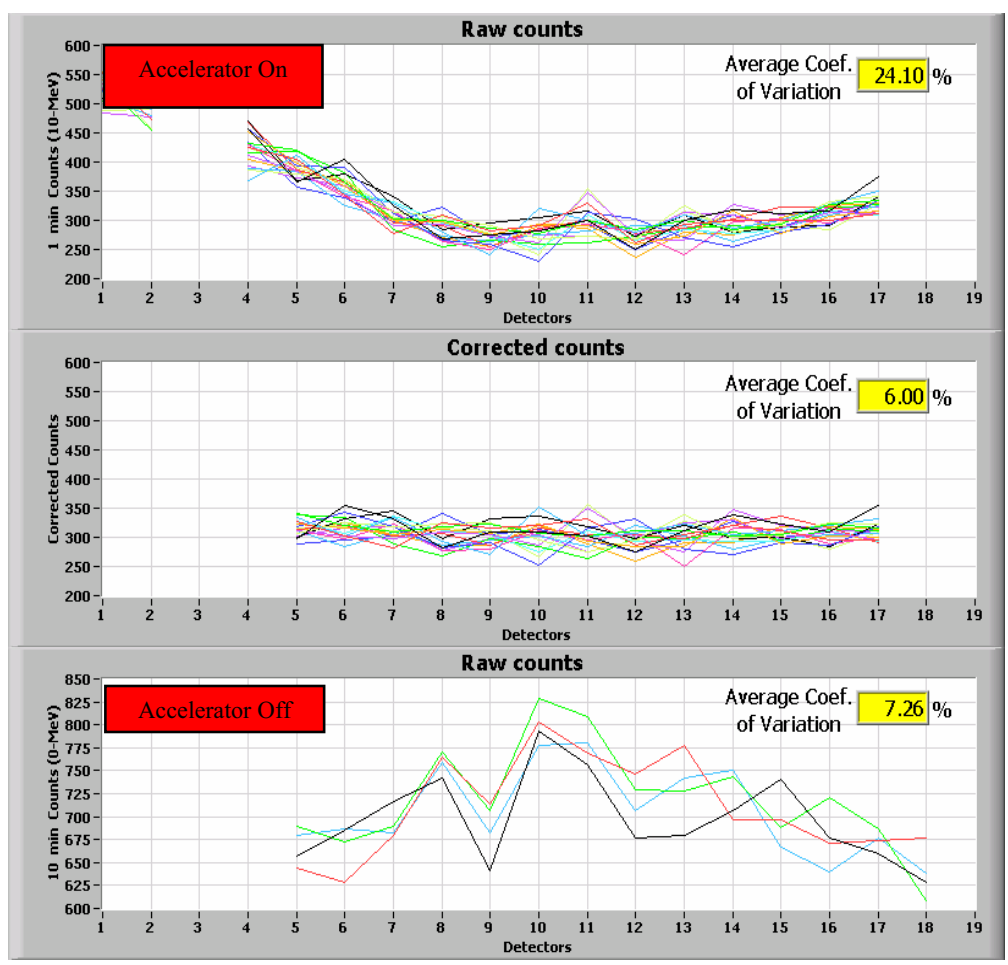

Figure C-1. GM detector raw data with accelerator ON (top), calculated correction factors (center), and raw data with accelerator OFF (bottom). 
Appendix D

\section{Testing Detector Normalizations with DU}




\section{Appendix D}

\section{Testing Detector Normalizations with DU}

The gamma correction factors previously calculated in ten-minute runs were applied to the acquisition system. Acquisition data was collected to test the correction factors. As noted earlier, it was not possible to correct detectors $\# 1, \# 2, \# 3$, and \#4 because they were not available at the time of data collection, however, these detectors have been corrected since that time. The accelerator was operated at $10 \mathrm{MeV}$ with the Polyethylene Calibration Pallet in the photon beam, center container position.

Windowing was the same as the calibration data set. Runs were ten minutes long. Figure D-1 displays the raw and corrected gamma counts. The three heavy lines represent runs where $4.8 \mathrm{~kg}$ of DU was present in the center of the Polyethylene Calibration Pallet. The heavy pink line is without the polyethylene plug [used to provide access to the center of each calibration pallet] in the pallet. Notice in Figure D-1(top) the runs without DU are relatively flat compared to the runs with DU. Figure D-2 (top and bottom) show the raw \& corrected PND counts. The data corresponds to the GM data. Notice when the shielding plug was out of the pallet, the DU was easily detected by PNDs $\# 2, \# 3$, \#4,\#5, and \#6 because these PNDs faced the plug opening. In all other cases, the DU was not detectable.

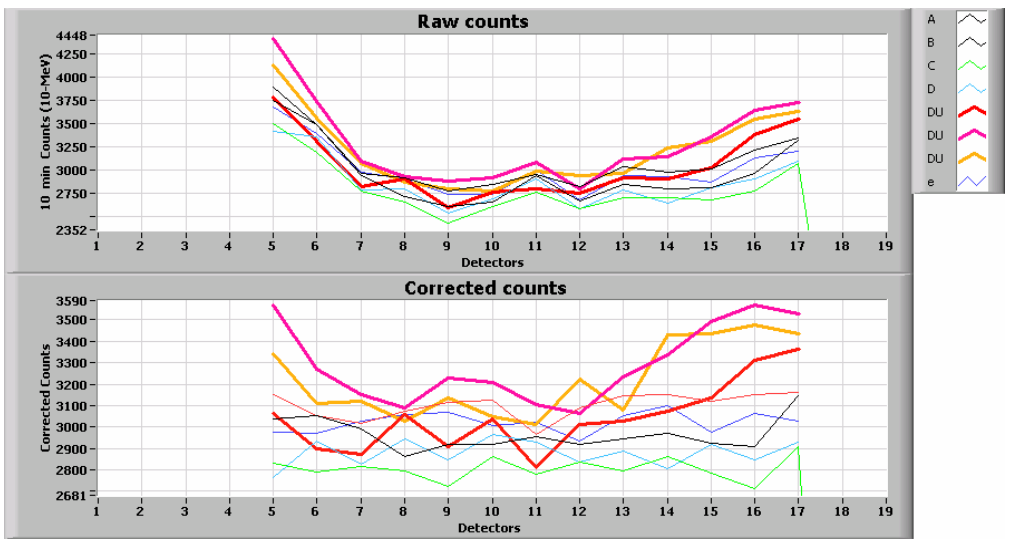

Figure D-1. Raw data (top) for GM detectors with and without DU and calculated correction factors (bottom) applied with and without DU.

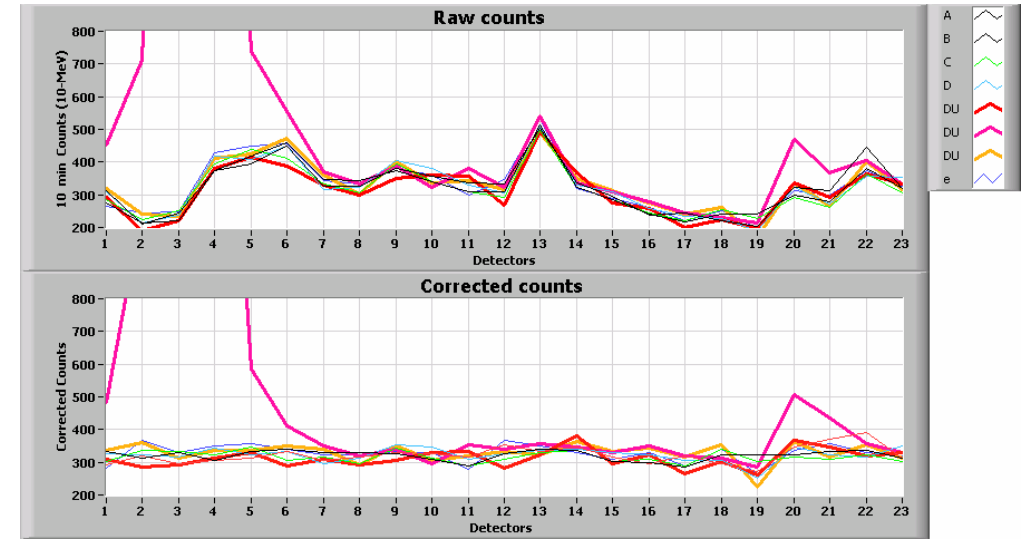

Figure D-2. Raw data (top) for PND detectors with and without DU and calculated correction factors (bottom) applied with and without DU. 